\title{
On the Linkage among Strong Stratospheric Mass Circulation, Stratospheric Sudden Warming, and Cold Weather Events ${ }^{\mathscr{O}}$
}

\author{
YUEYUE YU \\ Key Laboratory of Meteorological Disaster, Ministry of Education, and Joint International Research Laboratory \\ of Climate and Environment Change, and Collaborative Innovation Center on Forecast and Evaluation of \\ Meteorological Disasters, Nanjing University of Information Science and Technology, Nanjing, China \\ MING CAI \\ Department of Earth, Ocean and Atmospheric Sciences, Florida State University, Tallahassee, Florida

\section{CHUNHUA SHI} \\ Key Laboratory of Meteorological Disaster, Ministry of Education, and Joint International Research Laboratory \\ of Climate and Environment Change, and Collaborative Innovation Center on Forecast and Evaluation of \\ Meteorological Disasters, Nanjing University of Information Science and Technology, Nanjing, China

\section{RONGCAI REN} \\ Key Laboratory of Meteorological Disaster, Ministry of Education, and Joint International Research Laboratory of Climate \\ and Environment Change, and Collaborative Innovation Center on Forecast and Evaluation of Meteorological Disasters, \\ Nanjing University of Information Science and Technology, Nanjing, and State Key Laboratory of Numerical \\ Modeling for Atmospheric Sciences and Geophysical Fluid Dynamics, Institute of Atmospheric
} Physics, Chinese Academy of Sciences, Beijing, China

(Manuscript received 25 March 2018, in final form 21 June 2018)

\begin{abstract}
It is well established that sudden stratospheric warming (SSW) events tend to be accompanied by continentalscale, surface cold-air outbreaks (CAOs) in midlatitudes in boreal winter. However, SSW events occur at most one to two times per winter, whereas CAOs occur three to seven times over each of the North American and Eurasian continents. Using the ERA-Interim dataset for 37 winters (November-March) from 1979 to 2016, we reveal that SSW events correspond to a large-amplitude or long-lasting subset of pulse-like, anomalously strong, stratospheric mass circulation events. The anomalously strong, stratospheric mass circulation events (referred to as PULSE events) occur more than nine times in an average winter. The "displacement" versus "split" types of SSWs tend to correspond to the "wavenumber 1" versus "wavenumber 2" types of PULSEs, though the relationship between split-type SSWs and wavenumber-2-type PULSEs is weaker. Like SSW events, PULSEs also have a close relationship with CAOs. The robust relationship with CAOs still holds for the PULSE events not accompanied by SSW events. Using PULSE events, we determine that more than $70 \%$ of CAOs in the 37 winters occur in the week before and after a PULSE event, with a false alarm rate of CAO occurrence of about $25.7 \%$. SSW events, however, are associated with only about $5.7 \%$ of CAOs, with a false alarm rate of $21.7 \%$. Therefore, the linkage between individual continental-scale CAOs and PULSE events represents a more generalized relationship between the stratospheric circulation anomalies and surface weather. PULSE signals should also be considered as a potentially useful stratospheric indicator of the occurrence of individual CAO events.
\end{abstract}

Supplemental information related to this paper is available at the Journals Online website: https://doi.org/10.1175/MWR-D-180110.s1.

Corresponding author: Yueyue Yu, yyu4@fsu.edu

\section{Introduction}

Sudden stratospheric warming (SSW) is the most drastic dynamic process in the stratosphere, characterized by a decelerated or reversed westerly jet surrounding the polar vortex and a reversed temperature gradient between high and midlatitudes over the course 
of a few days. During major warming events, a remarkable two-way coupling between the stratosphere and troposphere has been well documented (Eliassen and Palm 1961; Matsuno 1970; Butchart et al. 1982; Kodera et al. 1990; Holton et al. 1995; Baldwin and Dunkerton 1999, 2001; Shepherd 2002; Kushner and Polvani 2004; Polvani and Waugh 2004; Limpasuvan et al. 2004, 2005; McDaniel and Black 2005; Haynes 2005; Garfinkel et al. 2010). Large regions in the midlatitudes tend to be anomalously cold during the 1-2 months after the central dates of SSW events (Thompson et al. 2002; Kenyon and Hegerl 2008; Tomassini et al. 2012). Kolstad et al. (2010) and Woo et al. (2015) found that cold anomalies tend to occur over the southeastern United States within 1-2 weeks after the central dates of SSW events but over Eurasia during the 2 weeks before. Mitchell et al. (2013), Kidston et al. (2015), and Lehtonen and Karpechko (2016) pointed out that the evolution of surface air temperature reported by Kolstad et al. (2010) often occurs in vortex displacement events. In splitting events, cold anomalies tend to occur over the midlatitudes of both North America and Eurasia in the 1-2 months around the central dates. Displacement events are mainly due to stronger wavenumber-1 waves, whereas splitting events are the result of stronger wave activities of wavenumber- 2 preceded by strengthened wavenumber-1 waves (Liberato et al. 2007; Martius et al. 2009; Castanheira and Barriopedro 2010; Kuttippurath and Nikulin 2012).

As operational models have overall useful prediction skill for the timing and structure of vortex breakup processes beyond 1-2 weeks (Christiansen 2005; Charlton and Polvani 2007; Yoden et al. 2014; Tripathi et al. 2015), although this is still dependent on the methods and initial conditions, the relationship between the surface air temperature anomaly pattern and the major SSW of the two types has great importance in practical applications for subseasonal forecasts of cold air outbreaks (CAOs) in the midlatitudes of the Northern Hemisphere in winter. However, major SSW events occur, at most, once in most winters, whereas CAOs occur several times per winter; thus, using SSW as an indicator of CAOs would result in "missing forecasts" of CAOs.

Recent studies have begun to notice another shortlived signal in the stratosphere, which has a life cycle of 10-20 days. This signal is the stratospheric "PULSE" events, defined as pulse-like rapid increases in the meridional mass transport across the polar circle in the stratosphere above $400 \mathrm{~K}$ (Cai et al. 2016) or extreme planetary wave heat flux events (Shaw and Perlwitz 2013, 2014). Tripathi et al. (2015) included such events, together with SSW and stratospheric final warming, in the extreme vortex events category. Some studies have investigated the stratosphere-troposphere coupling processes during extreme planetary wave heat flux events (Shaw and Perlwitz 2013, 2014; Tripathi et al. 2015). Cai et al. (2016) pointed out that a robust relationship with CAOs exists for PULSE events, not limited to extremely large-amplitude events. There is a higher probability of CAO occurrence over the midlatitude regions of Eurasia and North America within the 1-2 weeks before and after the peak dates of PULSE events, consistent with the relationship between CAOs and SSW events reported by Kolstad et al. (2010). Follow-up work by Yu et al. (2018b) classified PULSE events into different types, according to whether wavenumber- 1 or wavenumber- 2 waves were dominant, and pointed out that the specific spatiotemporal evolution of surface CAOs is dependent on the type of PULSE event. Cai et al. (2016) also emphasized that not only the slowly varying signals in the stratosphere, but also the PULSE signal, have a longer predictability limit than surface continental CAOs in operational numerical models; thus, the linkage between PULSE events and CAOs may open up a new opportunity for subseasonal forecasts of CAOs in a hybrid (dynamical plus statistical) framework.

The global meridional mass circulation was systematically documented by the pioneering work of Johnson (1989) and his collaborators and followed by many subsequent studies [e.g., Cai and Shin (2014), and references therein]. The meridional mass circulation can be regarded as a modified version of general Lagrangian meridional circulation (Andrews et al. 1987) using a quasi-conservative variable, potential temperature, as the vertical coordinate. The meridional mass circulation connects the tropics to the poles and the troposphere to the stratosphere via the poleward warm air branch in the upper troposphere and stratosphere and the equatorward cold branch in the lower troposphere. The net mass transport across the polar circle in the midlatitudes is driven by anomalously westward-tilting and largeamplitude waves with a deep structure (Johnson 1989). On the one hand, the meridional mass transport into the polar stratosphere, which was used to define PULSE events by Cai et al. (2016), tends to be coupled with the equatorward transport of polar air mass at lower levels (i.e., the lower branch of meridional mass circulation) in the midlatitudes, which is one of the physical causes of CAOs (Yu et al. 2015a,b). An investigation of the meridional mass transport into the polar stratosphere and its associated surface temperature anomalies could help us obtain a better understanding of the relationship between PULSE events and CAOs, which has not been satisfactorily revealed in previous studies. On the other 
hand, as ample evidence for the close relationship between SSW events and surface weather exists in the literature, it is critical to ask whether PULSE events can be used as a stratospheric indicator of CAOs and what the relationship is between these two short-lived signals in the stratosphere. A recent study (Yu et al. 2018a) reported that the stratospheric mass transport across the polar circle directly and quantitatively accounts for the contribution from all the dynamical processes to the polar pressure/height tendency, which is one of the main features of weak polar vortex events, including SSW events. Thus, the meridional mass transport into the polar stratosphere can be used to directly link these pulse-like signals in the stratospheric circulation to the well-known SSW events, as well as their weather impact at the surface.

This paper is organized as follows. Section 2 describes the data used in this study and defines indices for 1) the spatial span of cold surface temperature anomalies of different amplitudes in midlatitude regions of the North America and Eurasian continents and 2) meridional mass transport into the polar stratosphere by total waves and the wavenumber-1 and wavenumber- 2 components. We then introduce the central date and type of SSW events during 37 winters (November-March) in the period of 19792016 used in this study. In section 3, we describe the climatological features of PULSE events, including peak number, duration, type, average intensity, and maximum intensity during the 37 winters. Subsequently, in section 4, we examine the statistical relationship between PULSE and SSW events. In section 5, a comparison of the main features in the spatiotemporal evolutions of CAOs associated with PULSE and SSW events is made. The conclusions of the study are provided in section 6 .

\section{Data and methods}

\section{a. Data}

The data used in this study include daily surface air temperature (SAT), surface pressure $P_{s}$, surface meridional wind $v_{s}$, and three-dimensional air temperature $T$, meridional wind $v$, and zonal wind $u$ fields derived from the 6-hourly ERA-Interim data from January 1979 to December 2016 (ECMWF 2012; Simmons et al. 2007; Dee et al. 2011). The data fields are on $1.5^{\circ}$ latitude $\times$ $1.5^{\circ}$ longitude grids and 37 pressure levels from 1000 to $1 \mathrm{hPa}$. Three-dimensional and surface potential temperature $\left(\theta\right.$ and $\left.\theta_{s}\right)$ fields are derived from daily fields of $T$, SAT, and $P_{s}$. Wavenumber-1 and wavenumber-2 components of meridional wind velocities at pressure levels $\left(v_{1}\right.$ and $\left.v_{2}\right)$ and surface level $\left(v_{\mathrm{s} 1}\right.$ and $\left.v_{\mathrm{s} 2}\right)$ are obtained via decomposing $v$ and $v_{s}$ using the Fourier transform method.

\section{b. Cold area indices}

The daily anomaly field of SAT is obtained by subtracting the climatological annual cycle from the total SAT and removing the winter (November-March) mean to filter out the interannual variability, which might be dominated by phenomena like El NiñoSouthern Oscillation and the quasi-biennial oscillation (Thompson et al. 2002). The local standard deviation of the SAT anomaly is derived from the root-meansquare of the SAT anomaly at each grid point for each calendar day in winter. Then, we followed Cai (2003) and Yu et al. (2015a,b,c) and derived two sets of cold area indices as the percentage area occupied by the negative SAT anomaly below $-\alpha$ local standard deviation $(\alpha=0,0.5,1.0$, etc. $)$ for midlatitude North America $\left(30^{\circ}-60^{\circ} \mathrm{N}, 60^{\circ}-120^{\circ} \mathrm{W}\right)$ and midlatitude Eurasia $\left(30^{\circ}-60^{\circ} \mathrm{N}, 0^{\circ}-135^{\circ} \mathrm{E}\right)$, denoted as $\mathrm{CNA} \alpha$ and $\mathrm{CEA} \alpha$, respectively. Here, we only present results derived from $\alpha=0.5$, as the relationship between cold area indices and SSW and PULSE events is not highly sensitive to the choice of $\alpha$.

\section{c. Central date and type of major SSW events}

There is a large body of studies (Charlton and Polvani 2007; Cohen and Jones 2011; Mitchell et al. 2013; Seviour et al. 2013; Hu et al. 2014; Gómez-Escolar et al. 2014; Lehtonen and Karpechko 2016) documenting the central dates of major SSW events using various reanalysis datasets (e.g., NCEP-NCAR, ERA-40, and ERA-Interim) and definitions, such as the definition based on the zonal mean zonal wind along $60^{\circ} \mathrm{N}$ at $10 \mathrm{hPa}$ and the classification method based on absolute vorticity (Charlton and Polvani 2007; Gómez-Escolar et al. 2014; Lehtonen and Karpechko 2016), as well as the vortex-based definition by Mitchell et al. (2013) and Seviour et al. (2013). A few recent studies (Butler et al. 2015; Palmeiro et al. 2015; Lehtonen and Karpechko 2016) have comprehensively compared different definitions of SSW events and their types. The results show that the timing and type of SSW events can be considered comparable despite some discrepancies in the detected events.

In this study, we used the central dates and types (listed in Table 1; displacement type denoted as "D" and split type denoted as "S") of SSW events in the period from December 1979 to March 2016 documented by Lehtonen and Karpechko (2016), who investigated the surface response to different types of SSW events, and thus, our results can be compared with their work 
TABLE 1. The central dates of SSW events and their types (displacement type denoted as D and split type denoted as S), along with the period, central dates, intensity, duration, peak number, and types (W1, W1*, W2, W2*, and None) of the co-occurring PULSE events in the 37 winters (November-March) during the period 1979-2016.

\begin{tabular}{|c|c|c|c|c|c|c|c|c|c|}
\hline \multirow[b]{2}{*}{ No. } & \multicolumn{2}{|c|}{ Major SSW } & \multicolumn{7}{|c|}{ PULSE } \\
\hline & Type & $\begin{array}{c}\text { Central } \\
\text { dates }\end{array}$ & Period & $\begin{array}{c}\text { Central } \\
\text { dates }\end{array}$ & $\begin{array}{c}\text { Intensity } \\
\text { (avg) }\end{array}$ & $\begin{array}{l}\text { Intensity } \\
(\max )\end{array}$ & $\begin{array}{c}\text { Duration } \\
\text { (days) }\end{array}$ & $\begin{array}{c}\text { Peak } \\
\text { No. }\end{array}$ & $\begin{array}{c}\text { Wave } \\
\text { type }\end{array}$ \\
\hline 1 & $\mathrm{D}$ & 4 Mar 1981 & 25 Feb-4 Mar 1981 & 28 Feb 1981 & 7.22 & 12.02 & 7 & 1 & $\mathrm{~W} 1$ \\
\hline 2 & & 4 Dec 1981 & 29 Nov-4 Dec 1981 & 1 Dec 1981 & 6.93 & 9.92 & 6 & 1 & \\
\hline 3 & & 15 Dec 1998 & 27 Nov-15 Dec 1998 & 7 Dec 1998 & 4.71 & 8.91 & 19 & 3 & \\
\hline 4 & & 5 Jan 2004 & 28 Dec 2003-5 Jan 2004 & 2 Jan 2004 & 5.02 & 9.05 & 9 & 2 & \\
\hline 5 & & 22 Feb 2008 & 16-20 Feb 2008 & 18 Feb 2008 & 5.48 & 9.24 & 5 & 1 & \\
\hline 6 & & 1 Mar 1980 & 25 Feb-4 Mar 1980 & 27 Feb 1980 & 3.23 & 5.42 & 8 & 1 & $\mathrm{~W} 1 *$ \\
\hline 7 & & 24 Feb 1984 & 16-28 Feb 1984 & 20 Feb 1984 & 5.93 & 8.25 & 13 & 3 & \\
\hline 8 & & 23 Jan 1987 & 15-25 Jan 1987 & 23 Jan 1987 & 3.41 & 6.04 & 11 & 2 & \\
\hline 9 & & 17 Feb 2002 & 7-17 Feb 2002 & 11 Feb 2002 & 4.11 & 5.58 & 11 & 2 & \\
\hline 10 & & 24 Feb 2007 & 17 Jan-26 Feb 2007 & 21 Feb 2007 & 5.72 & 8.34 & 10 & 1 & \\
\hline 11 & & 20 Mar 2000 & 17-20 Mar 2000 & 18 Mar 2000 & 5.64 & 7.40 & 4 & 1 & $\mathrm{~W} 2$ \\
\hline 12 & & 30 Dec 2001 & 29 Dec 2001-1 Jan 2002 & 30 Dec 2001 & 3.93 & 5.67 & 4 & 1 & None \\
\hline 13 & & 21 Jan 2006 & & & & & - & & \\
\hline 14 & S & 1 Jan 1985 & 17 Dec 1984-2 Jan 1985 & 25 Dec 1984 & 5.52 & 9.99 & 17 & 3 & $\mathrm{~W} 2$ \\
\hline 15 & & 21 Feb 1989 & 6-25 Feb 1989 & 15 Feb 1989 & 5.91 & 14.10 & 20 & 3 & \\
\hline 16 & & 18 Jan 2003 & 16-19 Jan 2003 & 17 Jan 2003 & 4.76 & 6.14 & 4 & 1 & \\
\hline 17 & & 24 Jan 2009 & 12 Jan-11 Feb 2009 & 25 Jan 2009 & 5.98 & 13.50 & 31 & 5 & \\
\hline 18 & & 6 Jan 2013 & 2-18 Jan 2013 & 10 Jan 2013 & 5.34 & 8.57 & 17 & 2 & $\mathrm{~W} 2 *$ \\
\hline 19 & & 11 Feb 2001 & 24 Jan-19 Feb 2001 & 6 Feb 2001 & 4.58 & 10.06 & 27 & 4 & $\mathrm{~W} 1 *$ \\
\hline 20 & & 8 Dec 1987 & 24 Nov-10 Dec 1987 & 4 Dec 1987 & 4.76 & 7.49 & 17 & 3 & \\
\hline 21 & & 26 Jan 2010 & 17-29 Jan 2010 & 23 Jan 2010 & 5.21 & 8.20 & 13 & 2 & \\
\hline 22 & & 26 Feb 1999 & 23-27 Feb 1999 & 24 Feb 1999 & 5.87 & 7.87 & 5 & 1 & W1 \\
\hline 23 & & 14 Mar 1988 & & & - & & & & \\
\hline
\end{tabular}

directly. We plotted the daily zonal mean zonal wind velocity at $60^{\circ} \mathrm{N}$, together with the polar cap $\left(60^{\circ}-90^{\circ} \mathrm{N}\right)$ averaged temperature at the $10-\mathrm{hPa}$ level derived from the ERA-Interim data (not shown), to confirm that the dates of SSW events listed by Lehtonen and Karpechko (2016) of major warmings are exactly the same as the dates when the polar jet reversed direction, which is the most common definition of major SSW events (e.g., Charlton and Polvani 2007). As listed in Table 1, there are 23 major events during the 37 winters, of which 13 events are " $D$ " type, and the others are "S" type.

\section{d. Stratospheric mass transport indices and definition of PULSE events}

We follow Cai et al. (2016) and Yu et al. (2015a,b, 2018b) and define a set of stratospheric meridional mass transport indices. The daily fields of potential temperature and meridional wind at the surface and 37 pressure levels are first interpolated onto 200 equally spaced sigma $(\sigma)$ levels. Then, the air mass transported into the polar stratosphere above $400 \mathrm{~K} \mathrm{~s}^{-1}$ fulfilled by the total waves and wavenumber- 1 and wavenumber- 2 waves (denoted as ST60N, ST60N_W1, and ST60N_W2, respectively), as a function of time $t$, are derived as

$$
\begin{aligned}
\operatorname{ST60N}(t) & =\int_{0}^{2 \pi} \int_{0}^{1} m_{\sigma} \times v(\lambda, \phi, \sigma, t) \times H[\theta(\lambda, \phi, \sigma, t)-400 \mathrm{~K}] d \sigma R \cos \phi d \lambda, \\
\operatorname{ST60N} \_W 1(t) & =\int_{0}^{2 \pi} \int_{0}^{1} m_{\sigma} \times v_{1}(\lambda, \phi, \sigma, t) \times H[\theta(\lambda, \phi, \sigma, t)-400 \mathrm{~K}] d \sigma R \cos \phi d \lambda, \\
\text { ST60N_W2 }(t) & =\int_{0}^{2 \pi} \int_{0}^{1} m_{\sigma} \times v_{2}(\lambda, \phi, \sigma, t) \times H[\theta(\lambda, \phi, \sigma, t)-400 \mathrm{~K}] d \sigma R \cos \phi d \lambda,
\end{aligned}
$$

where $m_{\sigma}=(\Delta \sigma / g) P_{s}$, which is the air mass between two adjacent sigma surfaces per unit area. Parameter $g$ is the gravitational constant and $\Delta \sigma=1 / 200 ; \phi$ is $60^{\circ} \mathrm{N}$ and $\lambda$ is longitude; $R$ is the radius of Earth; and $H(x)$ is 
the Heaviside function, such that $H(x)=1$ for $x \geq 0$, and otherwise $H(x)=0$. Note that although the zonal integral of both $v_{1}$ and $v_{2}$ is equal to 0 by definition, the net mass transported by those wave components of meridional wind is nontrivial. This is because the air mass above $400 \mathrm{~K}$ varies at each grid box along the latitudinal band, which is a result of the westward-tilted structure of baroclinically amplifying waves [see details in Johnson (1989)].

The anomaly fields of ST60N, ST60N_W1, and ST60N_W2 were obtained via the same procedure used to derive the SAT anomaly. We applied a new noiseassisted data analysis method, ensemble empirical mode decomposition (EEMD; Huang et al. 1998, 1999; $\mathrm{Wu}$ et al. 2009), to the stratospheric mass circulation indices in each winter. This method has been shown to be quite versatile in a broad range of applications for extracting signals from data generated in noisy nonlinear and nonstationary processes (e.g., Huang and Shen 2005; Huang and Wu 2008; Ji et al. 2014). Here, we use the EEMD method as a filtering tool by removing the highest-frequency intrinsic mode function from the total fields of ST60N, ST60N_W1, and ST60N_W2. Via this process, fine- and synoptic-scale features of the meridional mass transport into the polar stratosphere can be retained, while large portions of white-noise-like signals are filtered out. For ease of reference, we continue to refer to the filtered anomaly fields as ST60N, ST60N_W1, and ST60N_W2 hereafter. For the same consideration, we also apply the filtering process on the aforementioned cold area indices.

Cai et al. (2016) defined a PULSE event as a period of stronger meridional mass transport into the polar stratosphere above its 70th percentile for at least $n$ consecutive days $(n=2,3, \ldots, 8)$. A recent study by $\mathrm{Yu}$ et al. (2018b), however, defined a PULSE event by the peak time when the ST60N index reaches a local maximum that exceeds 0.5 standard deviation of the ST60N (i.e., meridional mass transport into the polar stratosphere is above the climatology by at least $1.67 \times$ $\left.10^{9} \mathrm{~kg} \mathrm{~s}^{-1}\right)$. It is necessary to consider various factors when examining the relationship between PULSE events and continental-scale CAOs. As can be seen from the time series of the ST60N index (presented by black curves in the upper panels in Fig. 1), a period of continuously stronger ST60N can have several subpeaks and/or last for longer than 2 weeks, and the duration, exact peak time, and intensity during such a period can greatly affect the corresponding temporal evolutions of cold area indices (presented by black curves in the middle and lower panels in Fig. 1) relative to a PULSE event that we defined. Therefore, in this study, we combine the two definitions made in previous studies to define an anomalously strong, stratospheric mass circulation event, also referred to as a PULSE event, as a period when ST60N continuously exceeds its 70th percentile (i.e., $1.53 \times 10^{9} \mathrm{~kg} \mathrm{~s}^{-1}$, according to the black curve in Fig. 2a). This definition is quite similar to Cai et al.'s (2016), but, in addition, the times of local peaks found in the time series of ST60N in each winter are also detected, which are similar to the peak dates of PULSE events defined in Yu et al. (2018b), except that this definition of a PULSE event does not separate a longlasting event with more than one peak into independent events. The number of local peaks and the total duration (days) of each PULSE event are counted as well. The central date of a PULSE event starting at $t_{0}$ and ending at $t_{M}$ is defined as

$$
t_{c}=\frac{\sum_{t=t_{0}}^{t_{\mathrm{M}}} t(i)\left[\mathrm{ST} 60 \mathrm{~N}(t)-\mathrm{ST} 60 \mathrm{~N}^{70 \mathrm{th}}\right]}{\sum_{t=t_{0}}^{t_{\mathrm{M}}} \operatorname{ST} 60 \mathrm{~N}(t)-\mathrm{ST} 60 \mathrm{~N}^{70 \mathrm{th}}},
$$

namely, the average date weighted by the deviation of ST60N from its 70th percentile (denoted as ST60N ${ }^{70 t h}$ ), which is the threshold of a PULSE event. By definition, the central date would be largely determined by or shift toward the time of the occurrence of the largest peak for multipeak events. The intensity of a PULSE event is defined in two ways: the average and maximum values during the whole period of the PULSE event. Moreover, following Yu et al. (2018b), we divide the PULSE events into five categories: 1) "W1," during which the maximum of ST60N_W1 is above its 70th percentile $\left(1.54 \times 10^{9} \mathrm{~kg} \mathrm{~s}^{-1}\right.$, according to the red curve in Fig. 2a), while the maximum of ST60N_W2 is below its 70 th percentile $\left(1.00 \times 10^{9} \mathrm{~kg} \mathrm{~s}^{-1}\right.$, according to the blue curve in Fig. 2a); 2) "W2," during which the maximum of ST60N_W2 is above its 70th percentile, while the maximum of ST60N_W1 is not; 3) "W1*," during which the period maxima of both the ST60N_W1 and ST60N_W2 indices exceed their 70th percentiles, but the maximum value of ST60N_W1 is larger; 4) "W2*," during which the maxima of both the ST60N_W1 and ST60N_W2 indices exceed their 70th percentiles, but the maximum value of ST60N_W2 is larger; and 5) "None," when neither of the maximum values of ST60N_W1 and ST60N_ W2 during the PULSE event exceeds its 70th percentile.

For a PULSE event defined in this study, we have five parameters measuring its characteristics: peak number, duration, type, period mean intensity, and maximum intensity. In the following section, we examine the climatological features of PULSE events in terms of these five parameters to gain a comprehensive understanding of the PULSE events. 

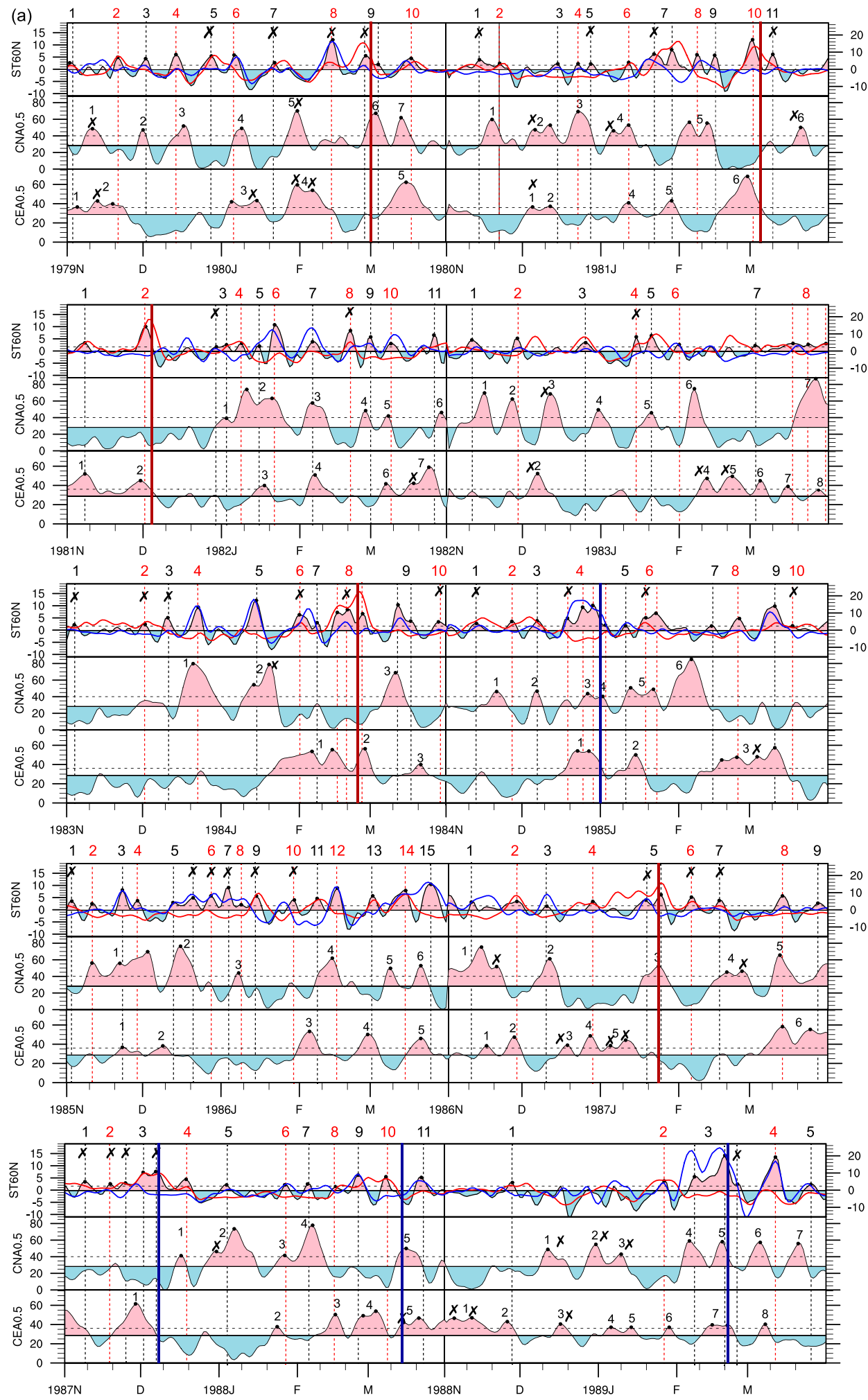

FIG. 1. (top) Time series of stratospheric mass circulation indices (black for ST60N, red for ST60N_W1, and blue for ST60N_W2), along with (middle) time series of cold area indices over midlatitude North America (CNA0.5) and (bottom) over midlatitude Eurasia (CEA0.5), in the winters from November 1979 to March 2016. The horizontal solid lines and dashed lines in each panel represent the 50th and 70th percentiles of ST60N, CNA0.5, and CEA0.5, respectively. The PULSE event, CAO event over North America (CAO_NA), 

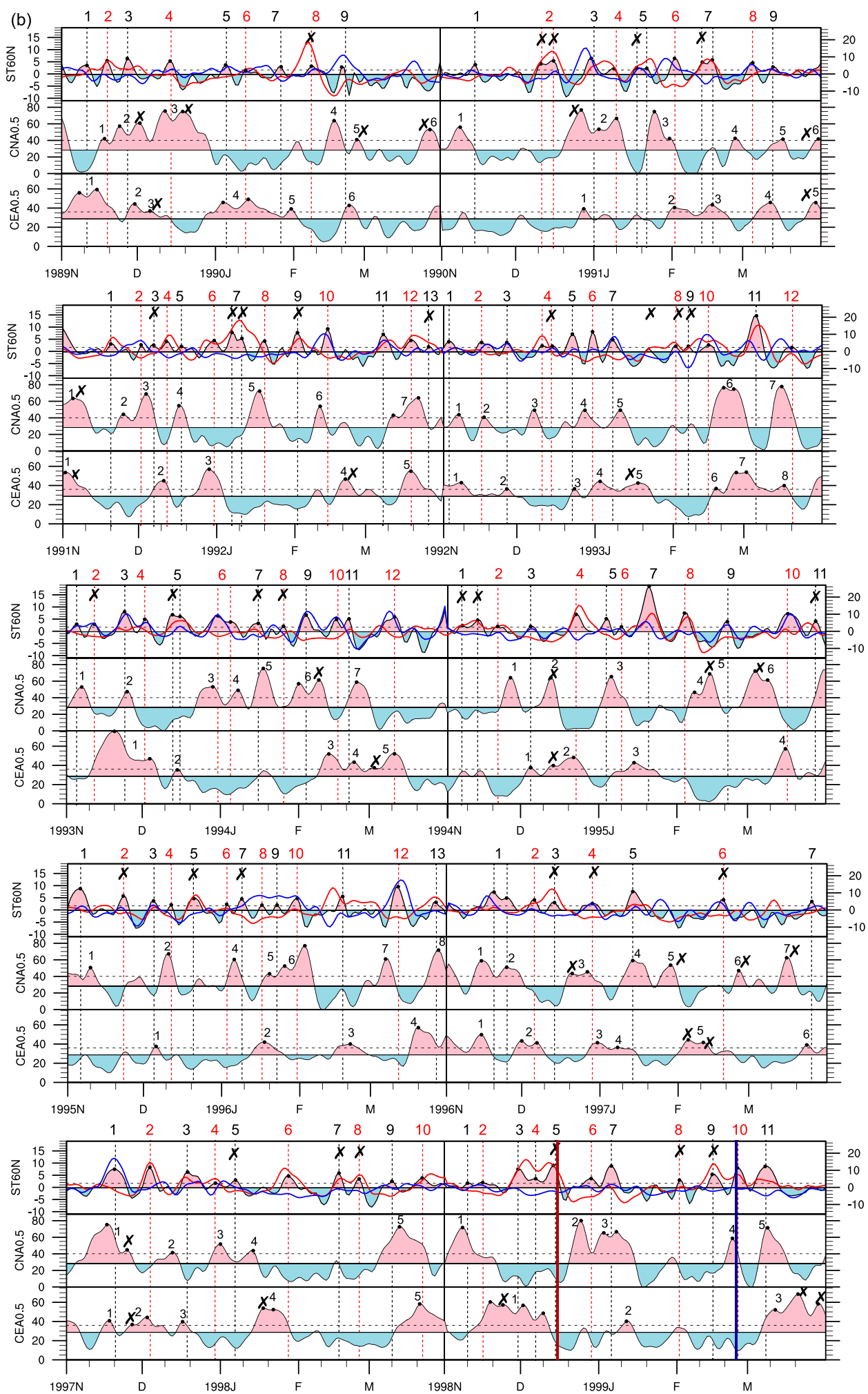

FIG. 1. (Continued) and CAO event over Eurasia are numbered starting from 1 for each winter. The central dates of D- and S-type SSW events, listed in Table 1, are marked by red and blue solid vertical lines, respectively. The CAO event peaks that were not paired with a PULSE event peak (allowing 7 days leadlag) are marked by a cross. The PULSE event peaks are highlighted by vertical dashed lines, and those that were not paired with a CAO event peak (allowing 7 days of lead-lag) are marked by a cross in the upper panels. 
(c)
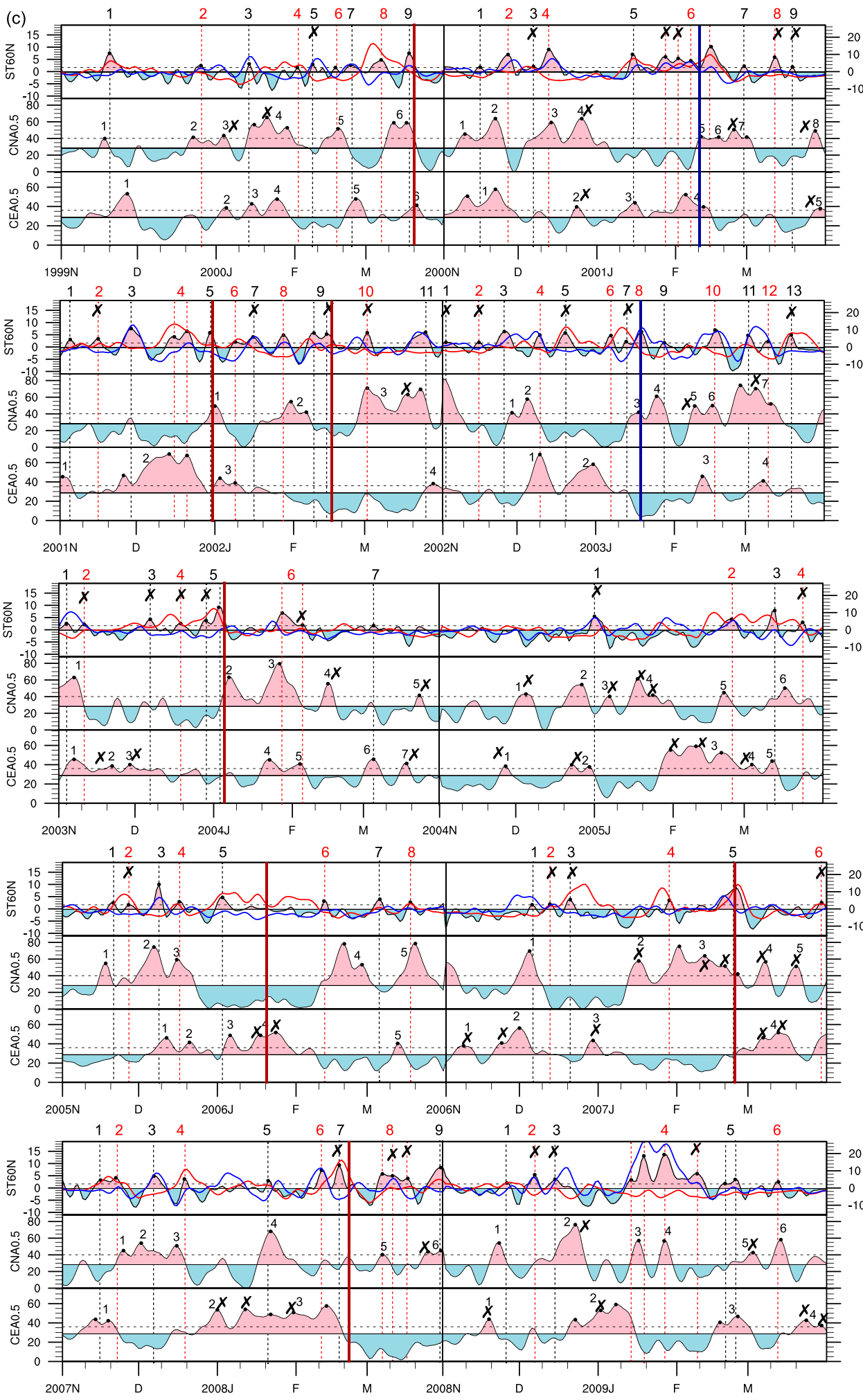

FIG. 1. (Continued) 

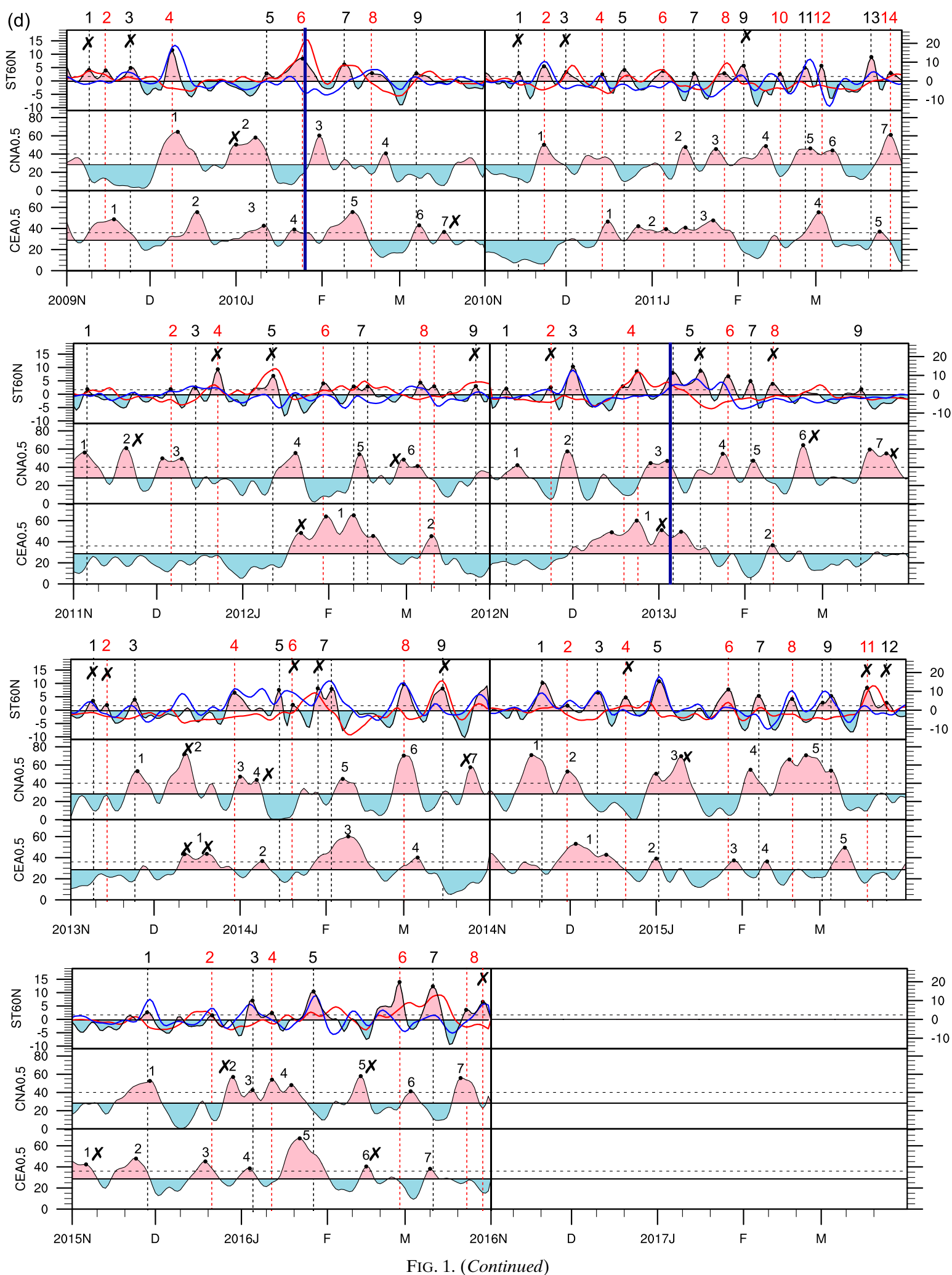

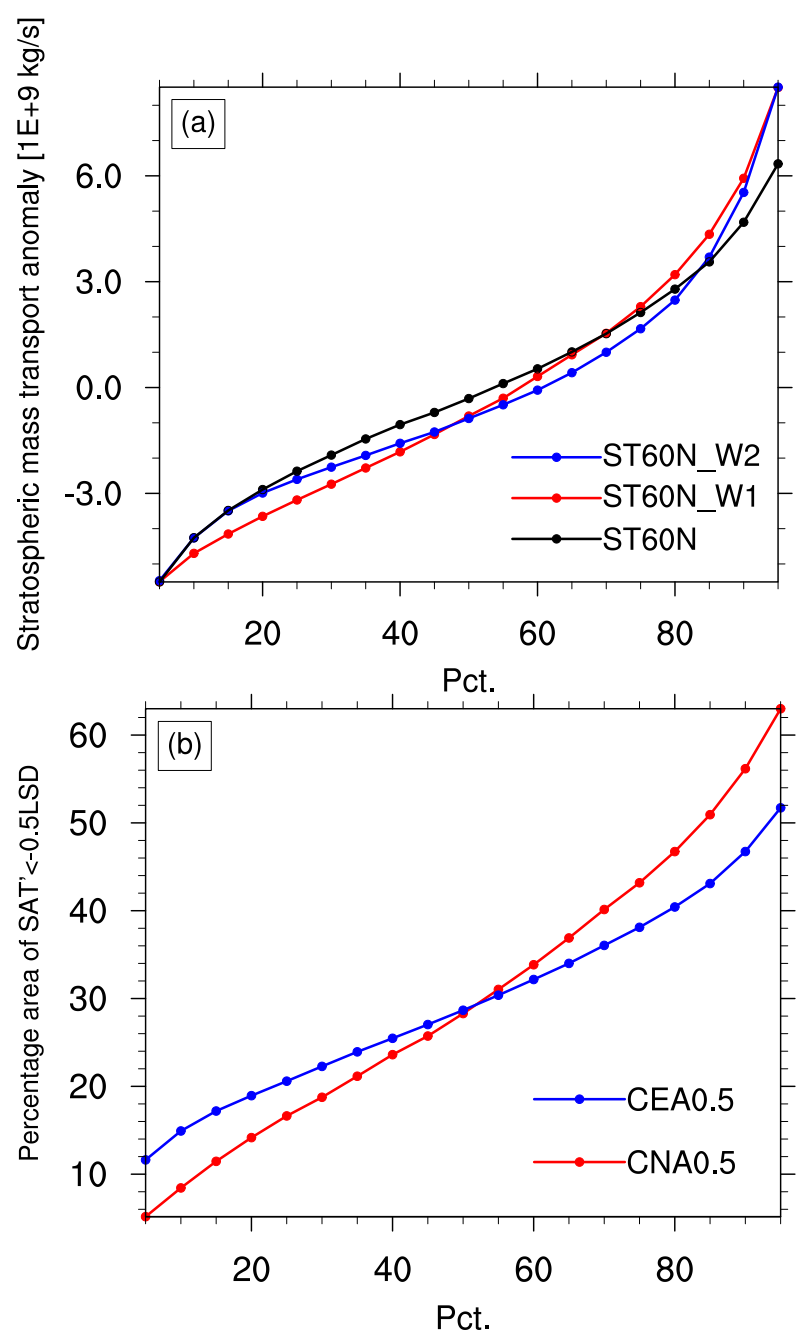

FIG. 2. Percentiles of (a) stratospheric mass circulation indices and (b) cold area indices over the midlatitudes of North America and Eurasia in the 37 winters (November-March) over the period 1979-2016.

\section{Climatological features of PULSE events}

In total, there are 346 PULSE events during the 37 winters (November-March) from 1979 to 2016 (i.e., more than nine PULSE events per winter). To provide statistical information on the climatological features of these PULSE events in winter, we subdivided the PULSE events into small intervals based on peak number, duration, type, and intensity parameters and then counted the probability of events falling into each of these intervals. The probability density functions (PDFs) of the PULSE events are given in Fig. 3. It can be seen that about $90 \%$ of PULSE events are single peaked. PULSE events with more than three peaks are quite rare. Although the longest PULSE event can last 31 days, most PULSE events have a duration of
3-5 days, consistent with the dominant 10-20-day time scale of the ST60N index reported by Yu et al. (2018a). PULSE events are mainly driven by stronger waves of wavenumber 1 and wavenumber 2 , indicated by the small percentage of PULSE events belonging to the None type. The occurrence probability of PULSE events with different ranges of average intensity is comparatively evenly distributed, despite a minimum value for the bin above the 95th percentile, which is about $5.2 \%$. As for the maximum intensity of PULSE events, more than $40 \%$ of events have a maximum ST60N value exceeding the 90th percentile, and about $23 \%$ have a maximum ST60N value exceeding the 95 th percentile.

To understand the interrelationship among the different parameters that measure the characteristics of PULSE events, we plot in Fig. 4 the joint PDFs of various combinations of two of the five parameters. It can be seen from Fig. 4a that PULSE events with more peaks tend to have longer durations. Single-peak PULSE events have a duration range of 1-10 days, double-peak PULSE events have a duration range of 5-17 days, and PULSE events with more than two peaks always last longer than 2 weeks. The linear correlation between the peak number and duration is 0.82 , confirming the direct relationship between the peak number and duration. As expected, the average intensity and maximum intensity are also positively correlated, as illustrated in Fig. 4j, and they have a near-perfect positive correlation (0.96). As seen from Figs. $4 c$ and $4 d$, singlepeak PULSE events have average and maximum intensities spread over all ranges from the 70th to 100th percentiles, but larger values of PDFs shift to smaller average intensity ranges. Double-peak PULSE events, in contrast, show larger PDF values in larger percentile bins for both average and maximum intensity. PULSE events with three peaks tend to have a narrow range of average intensity (i.e., 90th-95th percentiles) and maximum intensity (i.e., above 95th percentile). PULSE events with four and five peaks occur rarely, and their average and maximum intensities are in the 85th95th-percentile range and above the 90th percentile, respectively. Consistent with the large spread of intensity corresponding to the same peak number shown in Figs. $4 \mathrm{c}$ and $4 \mathrm{~d}$, the correlation of peak number with average intensity is 0.14 , and that with maximum intensity is slightly larger (0.26). The joint PDFs between duration and intensity in terms of average (Fig. 4f) and maximum (Fig. $4 \mathrm{~g}$ ) show a stronger in-phase relationship between duration and intensity. The positive correlations of duration with average intensity and maximum intensity are 0.52 and 0.62 , respectively. It can be said that in general, PULSE events with more 

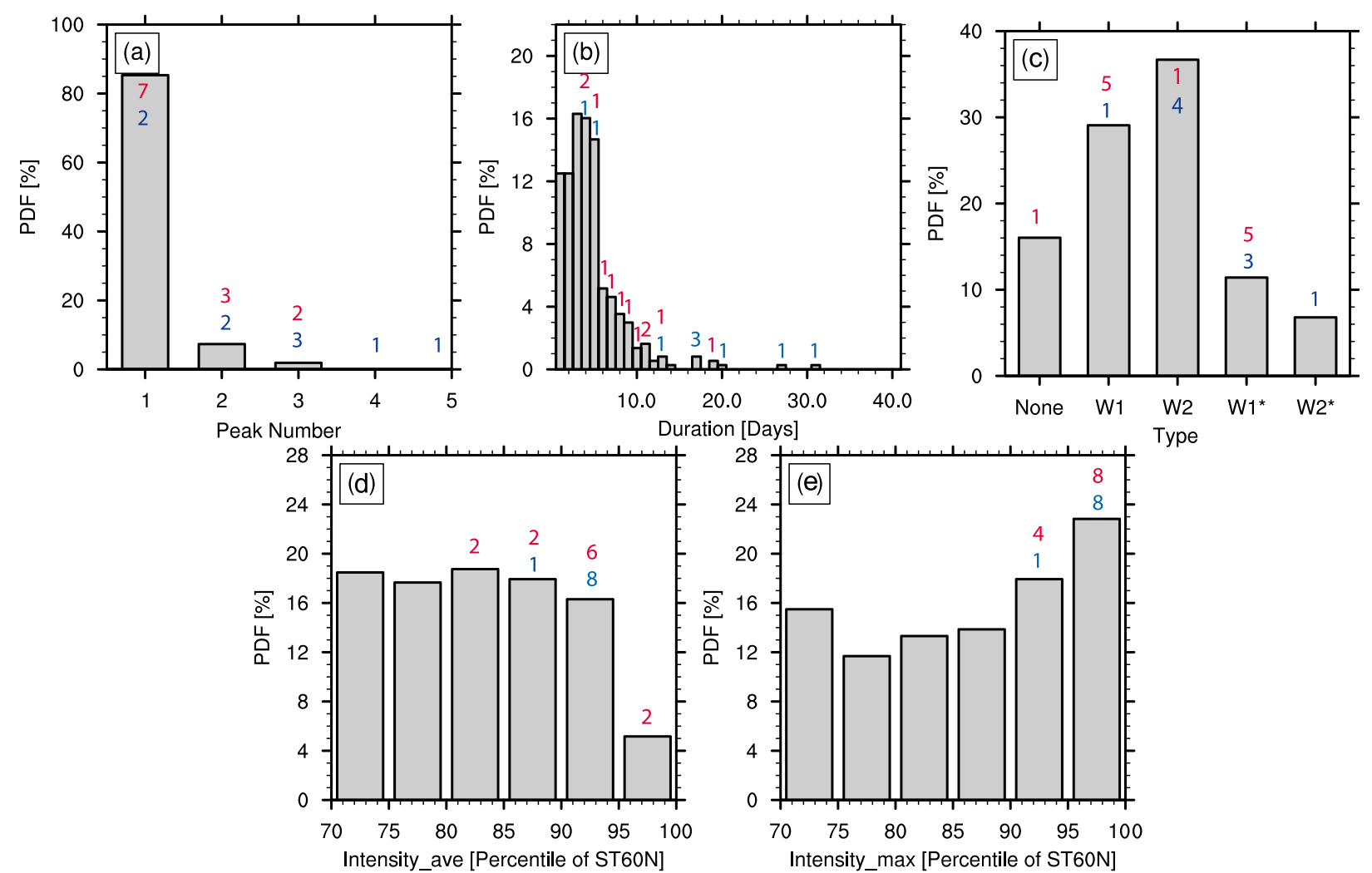

FIG. 3. PDF of (a) peak number, (b) duration, (c) type, (d) average intensity, and (e) maximum intensity of PULSE events in the 37 winters (November-March) over the period 1979-2016. Red and blue numbers indicate the number of PULSE events at each interval accompanied by D- and S-type SSW events, respectively.

peaks or longer duration tend to have larger intensity in terms of both average and maximum values of ST60N. Compared with average intensity, the maximum intensity is more closely related to the duration and peak number, and compared with peak number, the durations are more closely related to the intensity.

Let us then take a closer look at the joint PDFs between the type of event and the other parameters. The main feature found from the joint PDF of type with peak number (Fig. 4b) and duration (Fig. 4e) is that most of the None-type PULSE events are single-peak and shortlived (duration $<7$ days) events. Events with three or more peaks tend to belong to the W2- and W1*-type events, while most of the W1- and W2*-type events have a peak number up to two. Consistently, W2- and W1*-type events tend to last longer. As to the relationship between type and average intensity, we can see from Fig. 4h that the None-type PULSE events are relatively weak. W2-type events tend to be stronger, with a maximum PDF value around the 85th-90th percentiles, and the PDF value in the range above the 95th percentile is much larger than for the other types. The joint PDF of $\mathrm{W} 2 *$-type events in a lower range of intensity (70th-80th percentiles) is close to 0 . This intensity dependence on the type can be seen more clearly from the joint PDF of the maximum intensity, as shown in Fig. 4i, and is consistent with the statistical results provided by Yu et al. (2018b): as the PULSE intensity increases, the percentage of the types representing the dominance of wavenumber- 2 waves in driving the meridional mass transport into the polar stratosphere increases, while the percentage of the None types decreases.

Analyses above illustrate that the five parameters measuring the characteristics of PULSE events are interrelated, but only the relation between duration and peak number, and the relation between average and maximum intensity are robust. It can be inferred that when using a pulse event as an indicator of cold events, it is better to collect information of multiple features of the PULSE event to statistically construct an associated weather pattern.

\section{One-to-one correspondence between SSW and PULSE events}

Next, we examine the relationship between the newly proposed PULSE events and the SSW, which is 

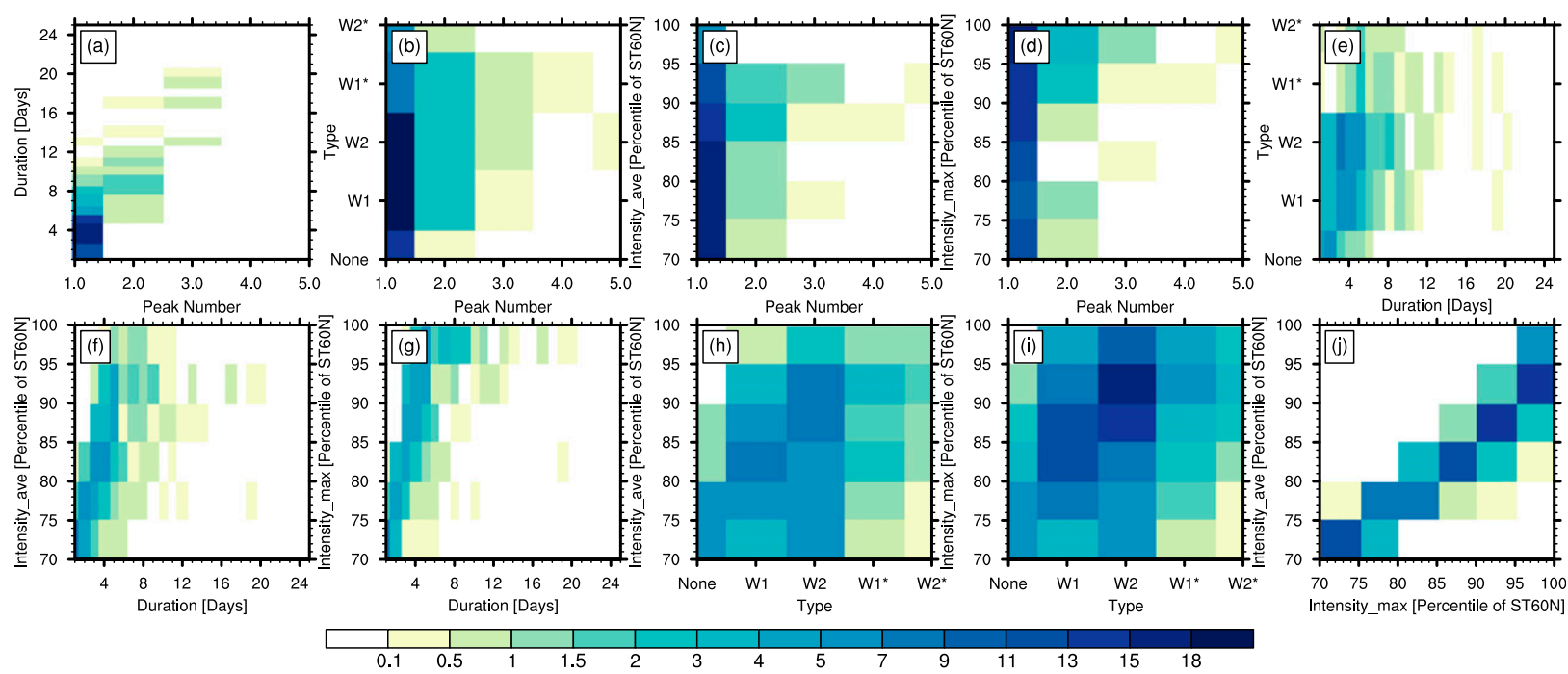

FIG. 4. Joint PDF of PULSE events in the 37 winters (November-March) over the period 1979-2016. (a) Peak number and duration, (b) peak number and type, (c) peak number and average intensity, (d) peak number and maximum intensity, (e) duration and type, (f) duration and average intensity, (g) duration and maximum intensity, (h) type and average intensity, (i) type and maximum intensity, and (j) maximum and average intensity.

considered a useful stratospheric indicator of continentalscale surface CAOs in midlatitudes in boreal winter. It can be seen from Fig. 1 that almost every SSW event co-occurs with one PULSE event. But, in turn, not every PULSE event, even the one with large amplitude, is accompanied by an SSW event. From Table 1, the oneto-one correspondence between PULSE and SSW events is clear. Out of $23 \mathrm{SSW}$ events, 21 were "simultaneously" accompanied by PULSE events, with the central dates of PULSE events leading most of the corresponding SSW events by a few days. The 21 SSWrelated PULSE events are all comparatively strong events, with an average intensity above the 80th percentile and maximum intensity above the 90th percentile of ST60N. As shown in Fig. 3, the PULSE events with an average intensity above the 80th percentile of the ST60N index account for about half of the total PULSE events, and PULSE events with a maximum intensity above the 90th percentile of ST60N account for $41 \%$ of all PULSE events (346 events in total), which occurred more frequently than SSW events. The occurrence of SSW events also exhibits a slight preference for PULSE events with longer durations. Among the 21 SSW-related PULSE events, none has a duration of fewer than 4 days, and two-thirds have a duration of longer than 7 days. Accordingly, SSW can be regarded as a special small subset of strong and relatively longlasting PULSE events.

There were still two SSW events (i.e., the D-type SSW event on 21 January 2006 and the S-type SSW event on 14 March 1988) that were not coupled with a PULSE event, which implies that although a strong PULSE event preferentially leads an SSW, the causal relationship is not simply between a single PULSE and an SSW event. Instead, an SSW event, which always corresponds to the negative phase of the stratospheric northern annular mode (NAM), is the polar mass accumulation effects of several consecutive PULSE events, which occur at a higher frequency, during a longer period (Cai et al. 2016; Yu et al. 2018a). According to Cai et al. (2016) and Yu et al. (2018a), each PULSE event transports more air mass into the polar stratosphere, effectively leading to an anomalous increase in the mass or pressure/height over the polar region and a decrease in the mass or pressure/height over the extratropics at stratospheric levels, while the diabatic cooling process plays a minor role. However, such an anomalous increase in polar stratospheric mass will be partly cancelled out by the anomalous decrease in polar stratospheric mass due to the following weaker mass circulation. Yu et al. (2018a) reported that the high-frequency variability at time scales of 10-20 days of meridional mass transport into the polar stratosphere (i.e., ST60N) is mainly due to rapid variations of vertically westward-tilting planetary waves. Only the low-frequency variability of stratospheric mass circulation, mainly driven by the wave amplitude's slow vacillation, plays the leading role in the quasi, $90^{\circ}$, out-of-phase lead relation of the meridional mass transport into the polar stratosphere with the polar stratospheric mass or negative NAM. This explains why not every PULSE event corresponds to an SSW event, but a strong and long-lasting PULSE event tends to 
occur with an SSW event. This also explains why an SSW event is not always associated with a PULSE event. For instance, an SSW event could be a result of mass accumulation by several PULSE events over a long period before the SSW event, but accompanied by a slightly above-normal mass transport event, which becomes the tipping point, as in the two SSW events that cooccurred with no PULSE event. This is why we refer to SSW events as a "special" subset of PULSE events when describing the relationship between SSW and PULSE events.

By marking in Fig. 3 the occurrence of PULSE events accompanied by D-type (denoted by red numbers) and S-type (denoted by blue numbers) SSW events according to their parameters, we found distinct features of PULSE events that correspond to different types of SSW events. Out of 12 PULSE events associated with D-type SSW events, seven are single-peak events, and the maximum peak number is three. PULSE events associated with S-type SSW events have a wider range of peak numbers, and only two out of nine are single-peak events. Furthermore, a large portion (six out of nine) of PULSE events associated with S-type SSW events have a duration longer than 2 weeks, while only one of the 12 PULSE events associated with D-type SSW events lasts that long. The average intensity of almost all (eight out of nine) PULSE events associated with S-type SSW events is between the 90th and 95th percentiles of the ST60N, whereas the average intensity of PULSE events associated with D-type SSW events has a wider range above the 80th percentile, although most (eight out of 12) are also in the range between the 90th and 95th percentiles. Similarly, the maximum intensity of almost all the PULSE events associated with S-type SSW events is above the 95th percentile, while four out of 12 of the PULSE events associated with D-type SSW events have maximum intensity in the range below. Therefore, from this one-to-one comparison between PULSE and SSW events, we infer that the PULSE events associated with S-type SSW events tend to have more peaks and longer duration and are slightly stronger than those associated with D-type SSW events.

According to previous studies on the spatial scale of the main wave forcing for the D- and S-type SSW events, as introduced above (Andrews et al. 1987; Liberato et al. 2007; Martius et al. 2009; Castanheira and Barriopedro 2010; Kuttippurath and Nikulin 2012), we may anticipate that the D-type SSW events correspond to W1-type PULSE events, while the S-type SSW events correspond to W2-type PULSE events, though the correspondence between S-type SSW events and W2-type PULSE events might be weaker. Table 1 shows that 10 out of 12 PULSE events associated with D-type SSW events belong to the $\mathrm{W} 1$ or $\mathrm{W} 1 *$ type, confirming the dominant role of strengthened wavenumber-1 waves for D-type SSW events. It can also be seen that there is one PULSE event accompanied by a D-type SSW event belonging to the None type, indicating that stronger waves of smaller spatial scales are also able to induce PULSE events cooccurring with D-type SSW events. Out of the nine S-type SSW events, five are accompanied by W2- or W2*-type PULSE events, while the remaining four events co-occurred with W1- or W1*-type PULSE events. In most cases of the $\mathrm{W} 2 *$ - and $\mathrm{W} 1 *$-type events associated with S-type SSW events, we found that stronger wavenumber- 1 waves tend to precede the strengthening of wavenumber- 2 waves, which is consistent with the findings of Martius et al. (2009), Nishii et al. (2011), and Bancalá et al. (2012). Despite the consistency pointed out above, we see clear differences between the classification method of SSW events based on the polar vortex structure and that of PULSE events based on the dominant spatial scale of wave forcing that drives the meridional mass transport of warm air into the polar stratosphere.

It is interesting to note that there seems to be some consistency and linkage among the following four findings: 1) the PULSE events associated with S-type SSW events tend to have more peaks and longer duration and are slightly stronger than those associated with D-type SSW events; 2) the PULSE events associated with S-type SSW events mainly belong to the W2 and W1* types; 3) W2- and W1*-type PULSE events tend to have more peaks and last longer; and 4) most of the PULSE events with large intensity belong to the W2 type. These findings again reveal the correspondence between SSWs of specific types and PULSE events of specific characteristics, confirming that SSW events are a special subset of PULSEs.

\section{Cold weather associated with SSW and PULSE events}

\section{a. Composite mean temporal evolution of cold area indices}

In this section, we compare the surface weather impact of SSW and PULSE events. Our focus is on the midlatitudes, where cold events exert the most severe impact. The black curves in Fig. 5 are the composite means of cold area indices, CNA0.5 (Figs. 5a,c) and CEA0.5 (Figs. 5b,d), associated with D-type (Figs. 5a,b) and S-type (Figs. 5c,d) SSW events. Recall that the CNA0.5 and CEA0.5 measure the percentage area occupied by SAT anomalies at least 0.5 local standard deviation below normal over the midlatitudes of the 


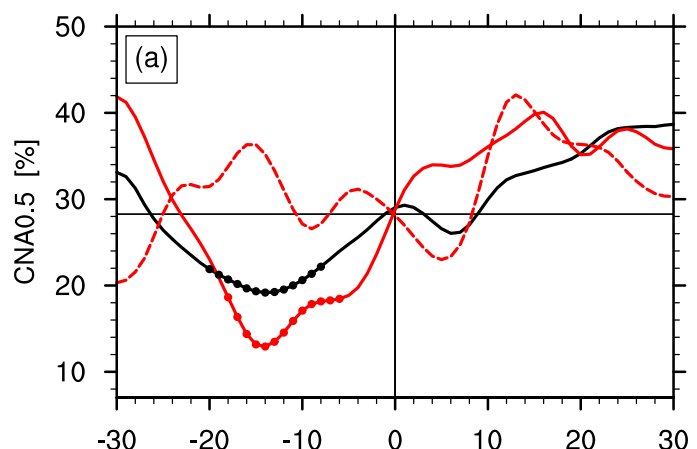

Lag Days from Central Dates of Type D SSW

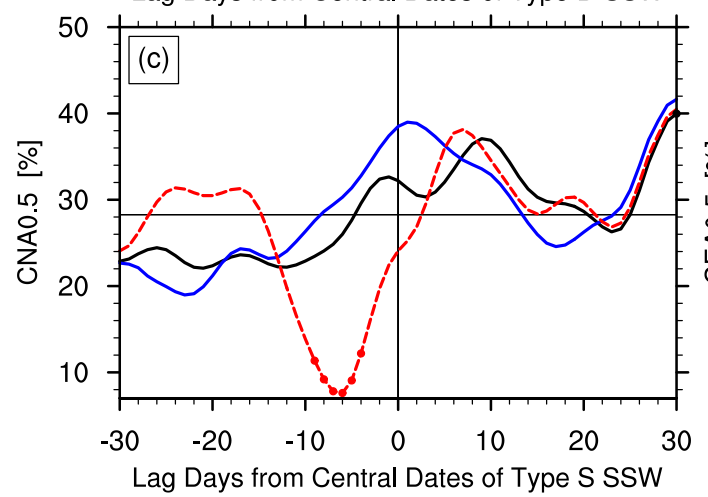

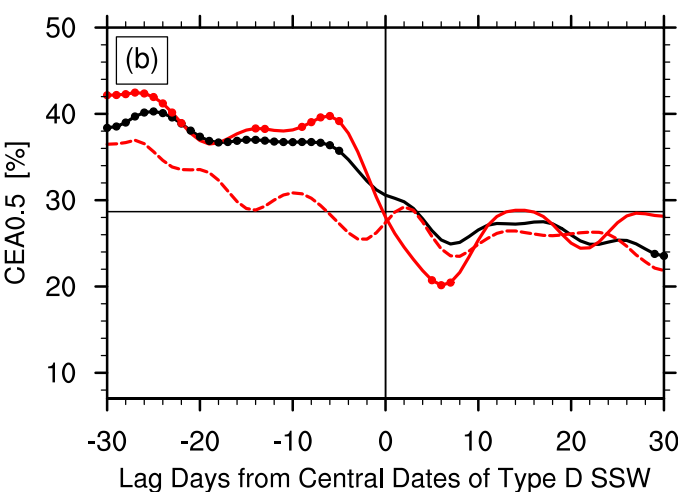

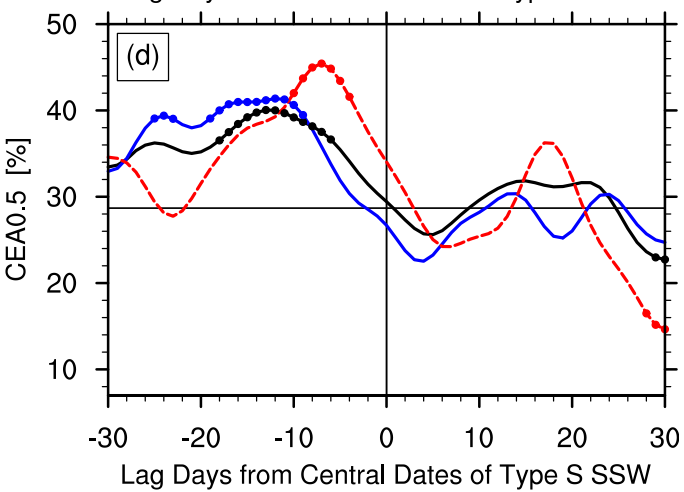

FIG. 5. (a),(c) Composite means of CNA0.5 (black curves; units: \%) and (b),(d) CEA0.5 (black curves; units: \%) at lag times from -30 to 30 days relative to the central dates of D- and S-type SSW events. Red solid curves, red dashed curves, and blue solid curves are the composite means of CNA0.5 and CEA0.5 relative to the central dates of SSW events accompanied by a PULSE event belonging to W1, W1*, and W2 types, respectively, according to Table 1. Composites associated with SSW events accompanied by a PULSE event belonging to W2* and None types are not shown, as the sample size for the composite analysis is less than two. The horizontal solid black line in each panel represents the 50th percentile of CNA0.5 and CEA0.5. Dots indicate composites above the $90 \%$ level of statistical significance.

North American and Eurasian continents, respectively, in the Northern Hemisphere. It can be seen that for both types of SSW event, the composite mean CNA0.5 is below its 50 th percentile ( $28.5 \%$; see Fig. 2 b), but the composite mean CEA 0.5 is significantly above its 50 th percentile (28.7\%; see Fig. 2b) in the month before the central dates of SSW events. Compared with S-type SSW events, during D-type SSW events, the composite mean value of CNA0.5 is much lower and statistically significant, and the statistically significant above-normal composite mean CEA0.5 lasts longer. After the central dates of D-type SSW events, the composite mean value of CNA0.5 rises above its 50th percentile, while that of the CEA0.5 falls below its 50th percentile. The composite mean value of CNA0.5 is generally above its 50th percentile in the 1-2 weeks and the fourth week following the central dates of S-type SSW events, with three peaks at lag times of $-1,10$, and 30 days after the peak dates of SSW events. The composite mean value of CEA0.5 rises a few percent above its 50th percentile in the 2-3 weeks after the central dates of S-type SSW events. The difference in the composite mean of cold area indices associated with SSW events between S and $\mathrm{D}$ types can be inferred by comparing the black curves in Fig. 5a with Fig. 5c and those in Fig. 5b with Fig. 5d. The composite mean CNA0.5 is larger in the 2-3 weeks centered on the central dates of S-type SSW events, compared with that associated with D-type SSW events. In the week before (after) that period (2-3 weeks centered on the peak dates), the CNA0.5 tends to be smaller (larger), while the CEA0.5 tends to be larger (smaller) in D-type SSW events. These features are consistent with those reported in Mitchell et al. (2013) and Lehtonen and Karpechko (2016), despite some minor disparities.

The difference in the relationships between D- and S-type SSW events and CAO over North America and Eurasia is related to the tropospheric circulation patterns associated with different types of SSW events. It is reported that blockings in the Euro-Atlantic sector 
mostly lead to the development of D-type warmings, whereas blockings in the Pacific region mostly precede S-type major SSWs (Martius et al. 2009; Nishii et al. 2011; Bancalá et al. 2012). The structure and evolution of the vortex after central dates of SSW differ greatly, which is responsible for differences in the following surface weather (Matthewman et al. 2009; Mitchell et al. 2011, 2013). The evolution of the anomalies during S-type events is more barotropic than during displacement events. As to the D-type SSW, increased blocking activity is observed over Canada in the month after, which might be the result of reflection of upwardpropagating planetary waves from Eurasia (Coughlin and Tung 2005; Kodera et al. 2008). Most of these previous studies pointed out that the difference in the associated tropospheric circulation anomalies with SSW events mainly depends on whether the SSW event is driven by stronger wavenumber 1 or wavenumber 2 .

It is also worth pointing out that the composites above the $90 \%$ statistical significance level are mainly found in limited periods, and those periods tend to be in the month before, rather than after, the central dates of SSW events. Moreover, the composite mean values of CNA0.5 during the period -30 to 30 days relative to the central dates of S-type SSW events are all statistically insignificant, indicating large case-to-case variability in the temporal variations of the cold area percentage over midlatitude North America around S-type SSW events. For this reason, we carefully divide D-type SSW events into five subgroups, according to the type of accompanying PULSE event. We only show the composite mean cold area indices in the 1 month before to 1 month after the central dates of D-type SSW events co-occurring with PULSE events of W1 (red solid curve) and W1* (red dashed curve) types in Fig. 5, as D-type SSW events tend to be accompanied by PULSE events with a duration belonging to the $\mathrm{W} 1$ or $\mathrm{W} 1 *$ type, and fewer than two D SSW events are accompanied by None-, W2*-, and W2-type PULSE events (see Table 1). It can be seen that the red solid curves are quite similar to the black curves, consistent with the fact that most of the D-type SSW events are accompanied by W1-type PULSE events. However, it is noteworthy that a large difference can be found between D-type SSW events belonging to the $\mathrm{W} 1$ and $\mathrm{W} 1 *$ subgroups in the composite mean temporal evolution of both CNA0.5 and CEA0.5. It can be seen that for SSW events accompanied by W1*-type PULSE events, the CNA0.5 is above the 50th percentile in two periods: one around -20 to -10 days before the central dates and the other in the $2-3$ weeks after the central dates of the SSW event. The variations in the composite mean CNA0.5 before the central dates of the SSW events belonging to the W1* subgroup are almost opposite to the variations associated with SSW events belonging to the W1 subgroup. The other minor difference is that the above-normal composite mean CEA0.5 in the month before the central dates is $5 \%-10 \%$ smaller for SSW events accompanied by W1*-type PULSE events than those accompanied by W1-type PULSE events. Similarly, we divide the S-type SSW events according to the type of accompanying PULSE event and examine the associated composite mean cold area indices. Again, only composites relative to the S-type SSW events belonging to the W2 (blue curve) and W1* subgroups (dashed red curve) that occur at least twice are shown in Figs. $5 \mathrm{c}$ and 5d. Owing to the dominance of the W2 type over the W1* type in the S-type SSW events, the black curves look close to the blue curves. The temporal evolution of the composite mean CNA0.5 associated with S-type SSW events belonging to the W1* subgroup, however, is quite similar to that associated with D-type SSW events belonging to the W1* subgroup (red dashed curve in Fig. 5a), both of which show two above-normal periods, namely, 3-4 weeks before and 2-3 weeks after central dates. This distinct temporal variation of CNA0.5 between the two subgroups of SSW events contributes to the uncertainty in the temporal evolution of cold area indices around the S-type SSW events. The temporal evolution of the composite mean CEA0.5 associated with S-type SSW events belonging to the W1* subgroup also shows an overall decreasing trend from 1 month before to 1 month after the central dates, as in D-type SSW events belonging to the W1* subgroup, even though the deviation from its 50th percentile is larger and there is a short period of CEA0.5 above the 50th percentile after the central dates, indicating possible modification by the different types of SSW on the relation of W1*-type PULSE events with Eurasian weather. These results, in general, indicate that the same type of SSW event classified based on the polar vortex structure can be related to different temporal variations in the large-scale cold events over the two continents. This difference may involve the various types of PULSE events co-occurring with SSW events.

Next, we investigate the relationship between cold area indices and PULSE events of each of the five types and how their relationship varies with peak number, duration, and intensity ranges of the PULSE events. We then compare this with the relationship between cold area indices and SSW events. As the None type accounts for a small portion of the PULSE events and the associated surface CAO pattern is highly variable from case to case according to Yu et al. (2018b), here, we only investigate the relationship between cold area indices and PULSE events belonging to the $\mathrm{W} 1, \mathrm{~W} 1 *$, W2, and $\mathrm{W} 2 *$ types. It is reported in section 3 that the duration 
has comparatively large positive correlation with peak number, average intensity, and maximum intensity; thus, key features of temporal evolution of cold area indices around PULSE events with different peak numbers and intensity ranges can be largely represented by the temporal evolution of cold area indices around PULSE events with different durations of each type. For this reason, we show in Fig. 6 only the temporal evolution of the composite mean CNA0.5 (left column) and CEA 0.5 (right column) from 1 month before to 1 month after the central dates of PULSE events with different ranges of duration and belonging to the W1 (first row), W1* (second row), W2 (third row), and W2* (last row) types, while the composite mean cold area indices around PULSE events of different peak numbers and intensity ranges are provided in Figs. S1-S3 in the online supplemental material.

Black curves in Fig. 6 indicate composite mean cold area indices relative to all PULSE events, and blue, yellow, and red curves indicate composites relative to PULSE events with durations of at least 4,6 , and 9 days, respectively. It can be seen that the temporal evolution of cold area indices associated with W1-type PULSE events is, overall, consistent with that associated with D-type SSW events accompanied by W1-type PULSE events (red solid curves in Figs. 5a,b), indicating cold events with a larger area of impact over the midlatitudes of the Eurasian continent before the central dates, but over midlatitude North America after the central dates. It has to be admitted that two disparities can be found in composite mean temporal evolution of CEA0.5 index between W1-type PULSE events and D-type SSW events accompanied by W1-type PULSE events: 1) the duration of above- and below-normal period before and after central dates is shorter in the former, and 2) as the duration increases, the peak time tends to shift to a later time, which is possibly due to the definition of central dates of PULSE events. The deviation of CEA0.5 and CNA0.5 from their 50th percentiles tends to increase as the duration of PULSE events lengthens, especially for CNA0.5. As D-type SSW events tend to be accompanied by PULSE events with a duration of at least 4 days, it is necessary to compare the blue, yellow, and red curves in Figs. $6 \mathrm{a}$ and $6 \mathrm{~b}$ with the red solid curves in Figs. $5 \mathrm{a}$ and $5 \mathrm{~b}$. Consistency between the two is found in terms of both temporal evolution and amplitude of the composites. The temporal evolution of the composite mean CEA0.5 around W1*-type PULSE events is similar to that around W1-type PULSE events. The composite mean CNA0.5, however, shows below-normal values in the 2 weeks centered on the central dates, but above-normal values in the period 10-20 days before and after the central dates. A comparison between the blue, yellow, and red curves in Figs. $6 \mathrm{c}$ and $6 \mathrm{~d}$ and the red dashed curves in Figs. 5a-d also shows high consistency between W1*-type PULSE events and SSW events of both types accompanied by W1* PULSE events despite a time shift of few days. Associated with W2-type PULSE events, the composite mean CNA0.5 is above its 50th percentile in the 2 weeks centered on the central dates of PULSE events, with a maximum value of $48 \%$ at day 0 , which is similar to the blue curve in Fig. 5c. It is interesting that even when considering all the W2-type PULSE events (121 events in total), the maximum value is still comparable to that in S-type SSW events, which means more than 100 W2-type PULSE events can be associated with cold events over North America. The composite mean values of CEA0.5 associated with W2-type PULSE events vary almost oppositely to W1-type PULSE events, with a smaller deviation from the 50th percentile. However, as the duration increases, the composite mean CEA0.5 tends to be above normal in the 2-4 weeks before the central dates, but slightly below normal after the central dates. This qualitatively resembles the composite mean CEA0.5 associated with S-type SSW events belonging to the W2 subgroup (blue curve in Fig. 5d), but the time shift can also be seen, and the above-normal values of CEA0.5 are much larger before SSW events than PULSE events, implying that the continental-scale CAO over Eurasia tends to be the prerequisite for the occurrence of S-type SSW events, but not for W2-type PULSE events. Associated with W2*-type PULSE events, the CNA0.5 does not show statistically significant composite features, whereas the composite mean CEA0.5 index is statistically higher during the period -20 to 10 days relative to the central dates.

The composite means of cold area indices associated with PULSE events of different peak numbers (Fig. S1) and different intensity ranges (Figs. S2, S3) share similar correspondence with the composite means of cold area indices associated with SSW events shown in Fig. 5. Slightly better or worse correspondence can be found dependent on types, which might be attributed to the complicated relationship between SSWs of S and D types and PULSE of five types with different ranges of duration, peak number, average intensity, and maximum intensity discussed in previous sections.

These results demonstrate that the relationship of cold area percentage over the midlatitudes of North America and Eurasia with SSW events follows the relationship of cold area indices with PULSE events, though a few disparities can be found (especially for the Eurasian continent). This also indicates that PULSE events are a more general indicator of CAOs than SSW events. 


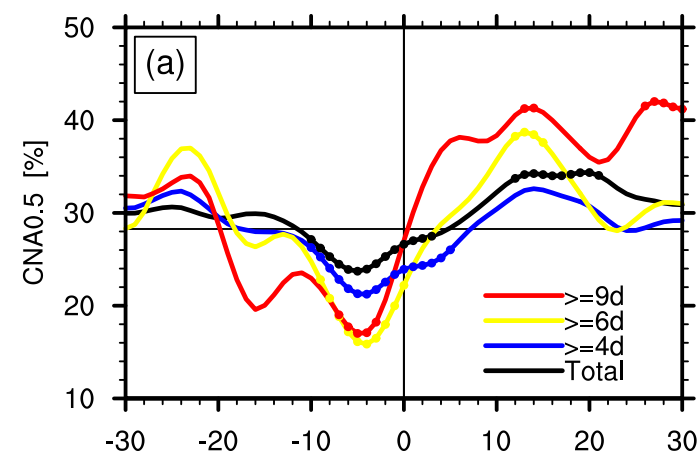

Lays from Central Dates of Type W1 PUISE

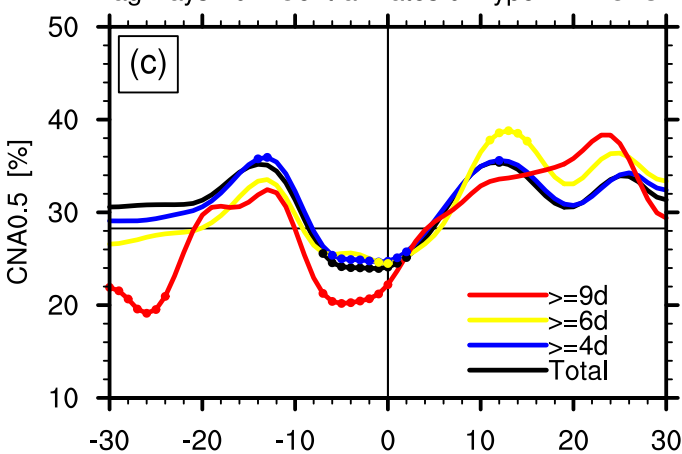

Lag Days from Central Dates of Type W1* PULSE

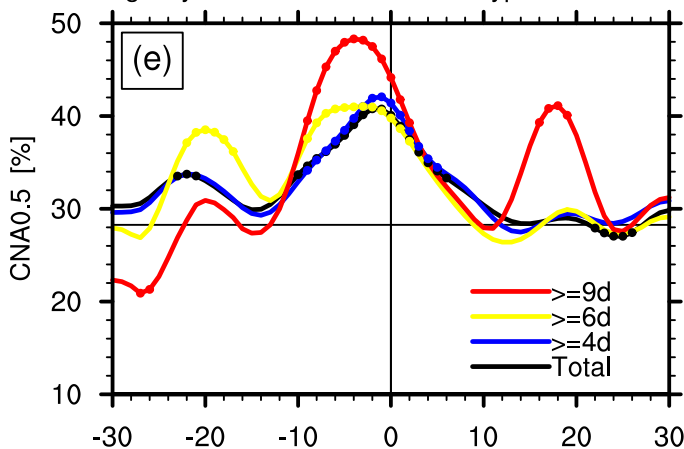

Lag Days from Central Dates of Type W2 PULSE

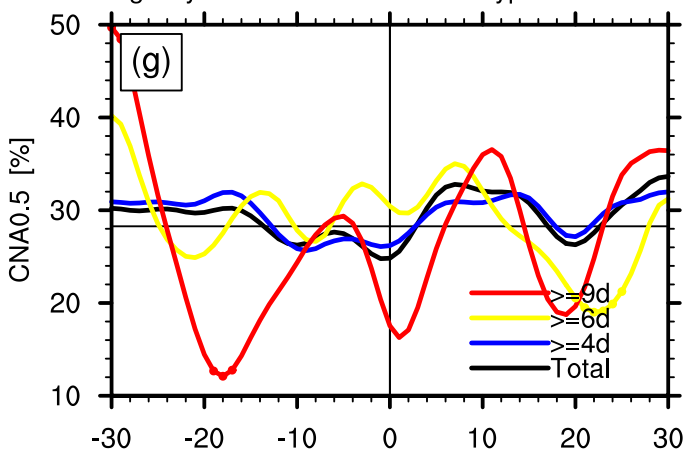

Lag Days from Central Dates of Type W2* PULSE
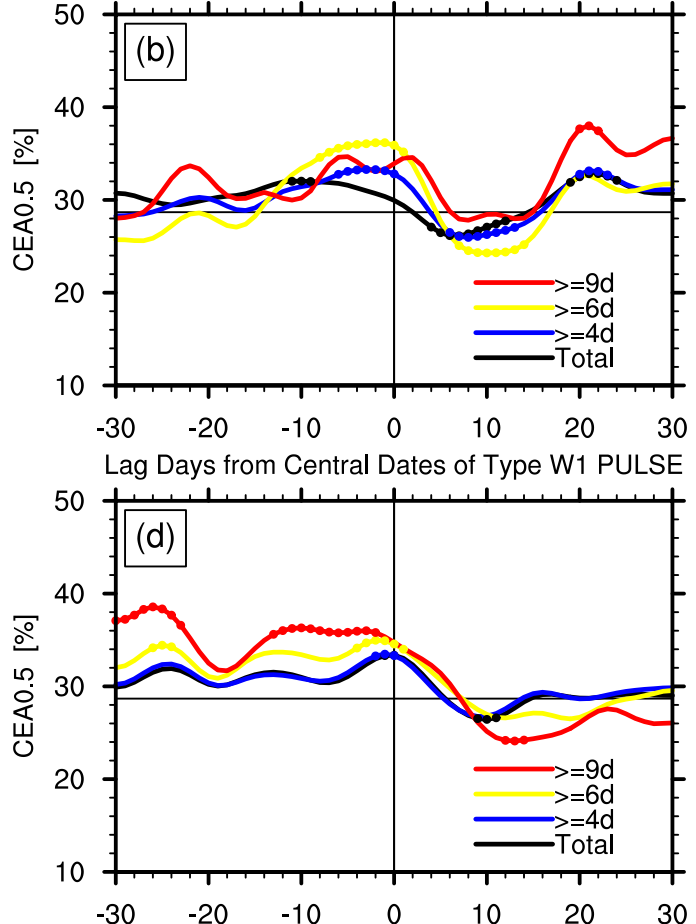

Lag Days from Central Dates of Type W1* PULSE
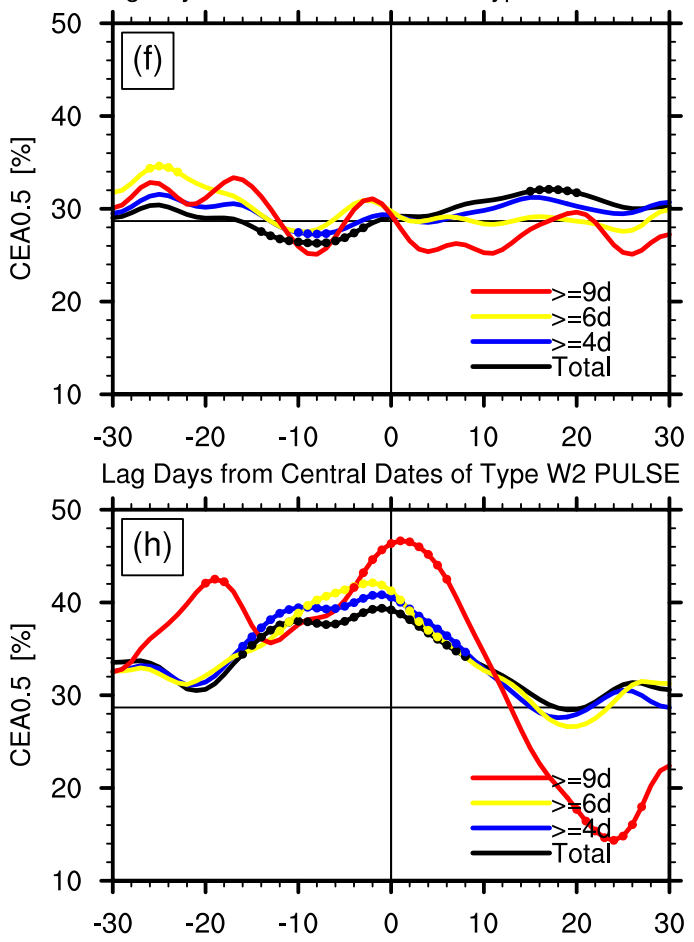

Lag Days from Central Dates of Type W2* PULSE

FIG. 6. Composite means of (left) CNA0.5 (units: \%) and (right) CEA0.5 (units: \%) at lag times from -30 to 30 days relative to the central dates of (a),(b) W1-; (c),(d) W1*-; (e),(f) W2-; and (g),(h) W2*-type PULSE events. Black curves indicate composites associated with all PULSE events, and PULSE events of duration $\geq 4,6$, and 9 days are indicated by blue, yellow, and red curves, respectively. The horizontal solid black line in each panel represents the 50th percentile of CNA0.5 and CEA0.5. Dots indicate composites above the $90 \%$ level of statistical significance. 
Another important point to be made here is that even when excluding those PULSE events accompanied by major SSW events, the robust relationship of CAOs with PULSE events still holds (not shown). Note that some PULSE events during winter (November-March) were also overlaid with less drastic polar vortex warming events (i.e., minor warming and final warming), which is a relatively rapid breakdown of the polar vortex termination each winter. We further excluded PULSE events corresponding to minor warming and final warming events and found that the relationship between CAOs and PULSE events remains almost the same. It can be inferred that the remaining frequently occurring PULSE events, as well as the PULSE events related to substantial polar warming events, which are widely known, should be considered for use as a stratospheric indicator of individual CAO events.

\section{b. One-to-one correspondence between $C A O$ events and PULSE and SSW events}

The temporal evolution of cold area indices shown above does not show the event-based correspondence of CAO events with PULSE and SSW events. Particularly for the frequently occurring PULSE events, larger values of cold area indices at longer lead-lag times might be due to another PULSE event in the neighborhood. It is necessary to diagnose how many PULSE events correspond to CAO events and how many PULSE events do not. This is similar to the sense of probability of detection (POD) and false alarm rate (FAR) of CAO events using PULSE and SSW events as a stratospheric circulation indicator. Here, a successful detection of $\mathrm{CAO}$ is defined when the peak of a CAO event occurs within a specific time window (e.g., -7 to +7 days) relative to the peak time of PULSE events or central dates of SSW events.

A CAO event is defined in a similar fashion to a PULSE event. It is a period when the cold area index continuously exceeds its 70 th percentile. The value of the 70th percentile is $40 \%$ for the CNA0.5 index and $36 \%$ for the CEA 0.5 index, as shown in Fig. 2b. During the 37 winters (November-March) in the period 19792016, there are 215 and 177 CAO events in total over the midlatitude regions of North America and Eurasia, respectively, and each of the CAO events is marked in the middle and lower panels of Fig. 1. It can be seen that although most CAO events are single peaked, there are some events with several local peaks, which is also the case for PULSE events. CAO events with more than one peak can be regarded as cold events during which the whole period is anomalously cold, with above-normal cold temperature coverage but several rounds of shortlived cold surges taking place during that cold period.
It can also be found from the time series of ST60N and cold area indices in Fig. 1 that there is a better correspondence between the peak of a CAO and the peak of a PULSE event, rather than their central dates.

Regarding this feature, we constructed a peakweighted correspondence calculating method to derive the probability of detection and false alarm rate of CAOs using PULSE events as an indicator. The first step is to pair PULSE event peaks with CAO peaks. We search for the PULSE event peak nearest to each CAO peak. If two or more peaks of the same CAO event or two or more peaks of different CAO events pair with the same PULSE peak, we pair the PULSE event with the CAO peak closest to the PULSE peak only and leave the other CAO peaks unpaired. We define a variable $\mathrm{Y} 1$ as a function of the event number of CAO events $n$ and peak number $k[k=1, K(n)]$, where $K(n)$ is the total number of peaks for CAO event $n$ and the lag days of the CAO peak relative to the paired PULSE peak $t_{\text {lag }}$. $\mathrm{Y} 1$ is set to 1 if the peak of the CAO is uniquely paired with a peak of the PULSE event; otherwise, Y1 is set to 0 . The POD of CAO using PULSE events as an indicator as a function of $t_{\text {lag }}$ is calculated according to

$$
\operatorname{POD}\left(t_{\mathrm{lag}}\right)=\frac{\sum_{n=1}^{N} \sum_{k=1}^{K} \mathrm{Y} 1\left(k, n, t_{\mathrm{lag}}\right) \mathrm{wgt}(k, n)}{N},
$$

where "wgt" is a weighting function derived as

$$
\operatorname{wgt}(k, n)=\frac{C^{\text {peak }}(k, n)-C^{70 \text { th }}}{\sum_{k=1}^{K(n)}\left[C^{\text {peak }}(k, n)-C^{70 \text { th }}\right]} .
$$

Here, $C$ corresponds to either CNA0.5 or CEA0.5; $C^{\text {peak }}(k, n)$ denotes the peak value of the cold area index (i.e., CNA0.5 or CEA0.5) for the peak $k$ of CAO event $n$; and $C^{70 t h}$ is the 70th percentile of the specific cold area index. If the CAO event $n$ is single peaked [i.e., $K(n)=$ $1]$, then "wgt" is equal to 1 . If the CAO event $n$ is multipeaked [i.e., $K(n)>1$ ], then "wgt" is larger for the peak with larger amplitude. Thus, the correspondence between a PULSE event peak and a CAO event peak with larger amplitude, which may exert more severe impacts on human life and the socioeconomic wellbeing of modern society, will be given more credit in counting the POD of the CAO event. By definition, if all the peaks of CAO events over the midlatitudes of the Eurasian or North American continents correspond uniquely to the peak of a PULSE event, then the sum of POD at all time lags is equal to 1 or $100 \%$. We then derive the FAR (i.e., the percentage of PULSE events not corresponding to a CAO peak) using the 
peak-weighted correspondence calculating method. We define a variable, Y2, as a function of the event number of PULSE events $n^{*}$ and peak number $k^{*}\left[k^{*}=1\right.$, $K^{*}\left(n^{*}\right)$ ], where $K^{*}\left(n^{*}\right)$ is the total number of peaks for PULSE event $n^{*}$ and the lag time of the PULSE peak relative to the paired CAO peak $t_{\text {lag }}$. Y2 is set to 1 if the PULSE event peak is uniquely paired with a CAO event peak; otherwise, $\mathrm{Y} 2$ is set to 0 .

The FAR of CAO events using PULSE events as an indicator as a function of $t_{\text {lag }}$ is derived as

$\operatorname{FAR}\left(t_{\text {lag }}\right)=\frac{\sum_{n^{*}=1}^{N^{*}} \sum_{k^{*}=1}^{K^{*}} \mathrm{Y} 2\left(k^{*}, n^{*}, t_{\text {lag }}\right) \operatorname{wgt}^{\prime}\left(k^{*}, n^{*}\right)}{N^{*}}$,

where

$$
\operatorname{wgt}^{\prime}\left(k^{*}, n^{*}\right)=\frac{\operatorname{ST} 60 \mathrm{~N}^{\text {peak }}\left(k^{*}, n^{*}\right)-\mathrm{ST}_{60 \mathrm{~N}^{70 t h}}}{\sum_{k^{*}=1}^{K^{*}(n)}\left[\operatorname{ST} 60 \mathrm{~N}^{\text {peak }}\left(k^{*}, n^{*}\right)-\mathrm{ST} 60 \mathrm{~N}^{70 \mathrm{th}}\right]} .
$$

Here, $\operatorname{ST} 60 \mathrm{~N}\left(k^{*}, n^{*}\right)$ denotes the peak value of ST60N index for the peak $k^{*}$ of PULSE event $n^{*}$, and ST60N ${ }^{70 t h}$ is the 70th percentile of the ST60N index. Accordingly, if the peak of a PULSE event with larger amplitude does not correspond to a CAO peak, it contributes more to the larger false alarm error using PULSE events as an indicator of $\mathrm{CAO}$ events.

The same analysis is conducted for CAO and SSW events, except there is no need to consider the multipeak situation. Listed in Table 2 are the accumulated POD and FAR values in the lead-lag time windows of -14 to 0,0 to $14,-7$ to $7,-5$ to 5 , and -3 to 3 days, relative to the central dates of PULSE and SSW events. It can be seen that the POD of CAO events over midlatitude North America that occurs in the 2-week period centered on the peak dates of PULSE events is $72.9 \%$ and is even larger for CAO events over midlatitude Eurasia, at $73.7 \%$. The POD for CAO events is much higher than the FAR $(25.7 \%)$. In other words, more than $70 \%$ of CAO peaks occur around PULSE peaks, and only $25 \%$ of PULSE events are not accompanied by CAOs. Narrowing down the time window, the POD of CAO events that occur over midlatitude North America in the 10-day period centered on the peak dates of PULSE events is $64.7 \%$ and is $65.5 \%$ for Eurasian CAO events, which is still larger than the FAR. The POD of CAO events that occur over midlatitude North America in the 1-week period centered on the peak dates of PULSE events is $50.8 \%$ and is $53.3 \%$ for Eurasian CAO events, which is also larger than the FAR $(41.6 \%)$. Considering the other two
TABLE 2. POD and FAR of using PULSE and SSW events as indicators of CAO events. A successful detection of a CAO peak is defined when the CAO event peak occurred within a specific time span relative to the peak time of PULSE events or central dates of SSW events. The time span for such statistics includes -14 to 0,0 to $14,-7$ to $+7,-5$ to +5 , and -3 to +3 days, relative to the peak time of PULSE events or central dates of SSW events. Values of POD without parentheses are related to CAOs over midlatitude regions of North America, while those in parentheses are related to CAOs over midlatitude Eurasia.

\begin{tabular}{cccccc}
\hline \hline \multirow{2}{*}{$\begin{array}{c}\text { Lag days } \\
\text { of CAO peaks }\end{array}$} & \multicolumn{2}{c}{ PULSE } & & \multicolumn{2}{c}{ SSW } \\
\cline { 2 - 3 } \cline { 5 - 6 } \cline { 5 - 6 } & POD & FAR & & POD & FAR \\
\hline-14 to 0 & $44.3 \%(47.0 \%)$ & $52.0 \%$ & & $2.92 \%(4.62 \%)$ & $26.1 \%$ \\
0 to +14 & $40.2 \%(45.1 \%)$ & $56.4 \%$ & & $4.67 \%(0.94 \%)$ & $34.8 \%$ \\
-7 to +7 & $72.9 \%(73.7 \%)$ & $25.7 \%$ & & $5.67 \%(5.13 \%)$ & $21.7 \%$ \\
-5 to +5 & $64.7 \%(65.5 \%)$ & $31.5 \%$ & & $4.78 \%(4.21 \%)$ & $21.9 \%$ \\
-3 to +3 & $50.8 \%(53.3 \%)$ & $41.6 \%$ & & $3.76 \%(2.27 \%)$ & $30.4 \%$ \\
\hline
\end{tabular}

windows, -14 to 0 and 0 to 14 days, the POD is smaller than the FAR, indicating that the occurrence of CAO events tends to be "simultaneous" with the PULSE events. Owing to the rare occurrence of SSW events, the POD of CAO events using SSW events as an indicator for all the time windows is less than $6 \%$. Note, however, that the FAR is not as small as expected, even though it is $100 \%$ certain that every SSW event can be paired with a CAO peak. This indicates that the SSW signal may not be a good indicator for the timing of occurrence of individual CAO events, despite it being useful for monthly mean surface air temperatures.

\section{Conclusions}

Using the ERA-Interim dataset covering 37 winters (November-March) in the period 1979-2016, we have examined the statistical relationship and further made a comparison in terms of the performance as a stratospheric indicator of winter cold air outbreak (CAO) events between two kinds of short-lived stratospheric signals: 1) SSW events that occur less than once in an average winter and 2) PULSE events, characterized by a pulse-like rapid increase in the meridional mass transport into the polar stratosphere, that occur more than nine times per winter. It is found that SSW events correspond to a small subset of large-amplitude and longlasting PULSE events. Vortex displacement (D type) SSW events tend to correspond to PULSE events dominated by stronger wavenumber-1 waves (W1 type and W1* type). Vortex splitting (S type) SSW events show a preference for being accompanied by PULSE events dominated by stronger wavenumber- 2 waves (W2), though a portion of S-type SSW events correspond to W1*-type PULSEs. The PULSE events that 
co-occur with S-type SSW events tend to have more peaks, last longer, and be stronger than those PULSE events that co-occur with D-type events, which shows some consistency with the dependence of peak number, duration, and intensity on the type of PULSE events.

The main features of the cold area percentage over the midlatitudes of North America and Eurasia related to SSW events are qualitatively consistent with previous studies. A larger area occupied by cold temperatures over midlatitude Eurasia tends to be found in the month before the central dates of SSW events of both types, while a larger area of cold temperatures over midlatitude North America tends to occur in the 3-4 weeks after the central dates of D-type SSW events and within the 2-3 weeks after central dates of S-type SSW events. In parallel with D- and S-type SSW events, a similar relationship with cold area indices is found mainly for W1- and W2-type PULSE events, though a few disparities can be found for the Eurasian continent. Note that W1*-type PULSE events co-occurred with eight SSW events of either S or D type. W1*-type PULSE events tend to be preceded by a larger cold area over both continents and followed by a larger cold area over midlatitude North America, which may increase the case-to-case variability of weather impacts associated with SSW events belonging to the same type (S or D). The relationship of PULSE events with cold area indices is found to be dependent on the peak number, duration, and average and maximum intensities. In general, PULSE events with larger peak number, longer duration, or larger intensity tend to have a more robust relationship with cold area indices. This robust relationship between PULSE events and cold area indices still holds when the PULSE events accompanied by major and minor SSW events, as well as final warming events, are excluded; thus, the remaining PULSE events can be attributed to CAO events during the 37 winters studied. In addition, to investigate the one-to-one correspondence between CAO events and PULSE and SSW events, we calculated the POD and FAR using PULSE or SSW events as indicators of CAO events. It was found that more than $70 \%$ of CAO events over the midlatitude regions of North America and Eurasia occur within the 2 weeks centered on the peak dates of PULSE events, while only $25 \%$ of PULSE events do not correspond to a CAO event peak. As for SSW events, a slightly smaller percentage of SSW events $(21.7 \%)$ did not correspond to CAOs in the 2 weeks centered on the peak dates; however, due to the rare occurrence of SSW, SSW events indicate only a very limited percentage of CAO events (less than 6\%).

For the purpose of improving the empirical prediction of subseasonal forecasts of CAOs, a useful indicator of
CAOs ought to satisfy three requirements. They should 1) vary at a frequency close to CAOs, which is important to minimize the miss rate; 2) have a robust relationship with CAOs, which is necessary to minimize the false alarm ratio; and 3) be predictable by operational models at a longer lead time. Based on whether PULSE events satisfy these requirements, we summarize below the evidence supporting the statement that PULSE signals could be a more generalized stratospheric indicator of individual CAOs than the widely known SSW events and a useful supplementary signal that should be considered in subseasonal forecasts of CAOs:

- In an average winter, PULSE events occur up to nine times, whereas SSW events occur less than once.

- Both PULSE and SSW events have a robust relationship with CAOs, though the SAT anomalies associated with SSW events tend to be larger.

- Both SSW and PULSE events are potentially predictable by operational models beyond the 2-week predictability limit of the troposphere (Christiansen 2005; Charlton and Polvani 2007; Yoden et al. 2014; Stan and Straus 2009; Li and Ding 2011; Zhang et al. 2013; Tripathi et al. 2015; Cai et al. 2016).

Note that except for the larger cold area over North America that occurred after PULSEs of W1 and W1* types, the cold periods over Eurasia lead the central dates of PULSE of these two types, and the cold periods over North America occurred in the meantime of PULSEs of W2 type. The boundary condition affected by surface temperature could be one of the causes of strengthened tropospheric planetary-scale waves (e.g., Kuroda and Kodera 1999; Garfinkel et al. 2010). And those waves with deep vertical structure would extend to the stratosphere, driving PULSEs. One may doubt if such a "simultaneous" relationship between PULSEs and CAOs is useful since the stratosphere seems to be a reflection rather than a cause or precursor with lead time information. Cai et al. (2016) reported that the pulse signals in the stratospheric mass circulation have a longer predictability limit than surface continental cold air outbreaks, up to 1 month in advance in winter 2013/14. Thus, as long as the model is capable of predicting the timing and intensity of the PULSEs beyond 2 weeks, using the simultaneous relationship of PULSEs and CAOs can still extend the predictability of CAOs in the hybrid (dynamical plus statistical) framework. To validate the longer predictability limit of PULSE, we are currently conducting an evaluation of the prediction skill of the CFSv2 forecasts for the timing and type of PULSEs using more years of available S2S forecast data, and the physical mechanism for the longer predictability will be investigated in the future work. 
Acknowledgments. This work was supported by grants from the National Science Foundation of China (41705039, 41575041, and 91537213), the Startup Foundation for Introducing Talent of NUIST (2017r068), and the Priority Academic Program Development of Jiangsu Higher Education Institutions (PAPD). The ERAInterim datasets used in this work are available from the ECMWF (http://www.ecmwf.int).

\section{REFERENCES}

Andrews, D., F. Taylor, and M. McIntyre, 1987: The influence of atmospheric waves on the general circulation of the middle atmosphere. Phil. Trans. Roy. Soc. London, 323A, 693-705, https://doi.org/10.1098/rsta.1987.0115.

Baldwin, M. P., and T. J. Dunkerton, 1999: Propagation of the Arctic Oscillation from the stratosphere to the troposphere. J. Geophys. Res., 104, 30 937-30 946, https://doi.org/10.1029/ 1999JD900445.

- , and —, 2001: Stratospheric harbingers of anomalous weather regimes. Science, 294, 581-584, https://doi.org/ 10.1126/science.1063315.

Bancalá, S., K. Krüger, and M. Giorgetta, 2012: The preconditioning of major sudden stratospheric warmings. J. Geophys. Res., 117, D04101, https://doi.org/10.1029/2011JD016769.

Butchart, N., S. A. Clough, T. N. Palmer, and P. J. Trevelyan, 1982: Simulations of an observed stratospheric warming with quasigeostrophic refractive index as a model diagnostic. Quart. J. Roy. Meteor. Soc., 108, 475-502, https://doi.org/10.1002/ qj. 49710845702.

Butler, A. H., D. J. Seidel, S. C. Hardiman, N. Butchart, T. Birner, and A. Match, 2015: Defining sudden stratospheric warmings. Bull. Amer. Meteor. Soc., 96, 1913-1928, https://doi.org/ 10.1175/BAMS-D-13-00173.1.

Cai, M., 2003: Potential vorticity intrusion index and climate variability of surface temperature. Geophys. Res. Lett., 30, 1119, https://doi.org/10.1029/2002GL015926.

— mass and angular momentum circulations: Boreal winter mean state. J. Atmos. Sci., 71, 2244-2263, https://doi.org/10.1175/ JAS-D-13-0175.1.

—, Y.-Y. Yu, Y. Deng, H. M. van den Dool, R. Ren, S. Saha, $\mathrm{X}$. Wu, and J. Huang, 2016: Feeling the pulse of the stratosphere: An emerging opportunity for predicting continentalscale cold-air outbreaks 1 month in advance. Bull. Amer. Meteor. Soc., 97, 1475-1489, https://doi.org/10.1175/BAMS-D14-00287.1.

Castanheira, J. M., and D. Barriopedro, 2010: Dynamical connection between tropospheric blockings and stratospheric polar vortex. Geophys. Res. Lett., 37, L13809, https://doi.org/ 10.1029/2010GL043819.

Charlton, A. J., and L. M. Polvani, 2007: A new look at stratospheric sudden warmings. Part I: Climatology and modeling benchmarks. J. Climate, 20, 449-469, https://doi.org/10.1175/ JCLI3996.1.

Christiansen, B., 2005: Downward propagation and statistical forecast of the near-surface weather. J. Geophys. Res., 110, D14104, https://doi.org/10.1029/2004JD005431.

Cohen, J., and J. Jones, 2011: Tropospheric precursors and stratospheric warmings. J. Climate, 24, 6562-6572, https://doi.org/ 10.1175/2011JCLI4160.1.
Coughlin, K., and K. K. Tung, 2005: Tropospheric wave response to decelerated stratosphere seen as downward propagation in northern annular mode. J. Geophys. Res., 110, D01103, https:// doi.org/10.1029/2004JD004661.

Dee, D. P., and Coauthors, 2011: The ERA-Interim reanalysis: Configuration and performance of the data assimilation system. Quart. J. Roy. Meteor. Soc., 137, 553-597, https://doi.org/ 10.1002/qj.828.

ECMWF, 2012: ERA Interim, daily: 1 November 1979-28 February 2011. European Centre for Medium-Range Weather Forecasts, accessed 1 July 2012, http://apps.ecmwf.int/ datasets/data/interim-full-daily/.

Eliassen, A. N., and E. Palm, 1961: On the transfer of energy in stationary mountain wave. Geofys. Publ., 22, 1-23.

Garfinkel, C. I., D. L. Hartmann, and F. Sassi, 2010: Tropospheric precursors of anomalous Northern Hemisphere stratospheric polar vortices. J. Climate, 23, 3282-3299, https://doi.org/ 10.1175/2010JCLI3010.1.

Gómez-Escolar, M., N. Calvo, D. Barriopedro, and S. Fueglistaler, 2014: Tropical response to stratospheric sudden warmings and its modulation by the QBO. J. Geophys. Res. Atmos., 119, 7382-7395, https://doi.org/10.1002/2013JD020560.

Haynes, P., 2005: Stratospheric dynamics. Annu. Rev. Fluid Mech., 37, 263-293, https://doi.org/10.1146/annurev.fluid.37.061903.175710.

Holton, J. R., P. H. Haynes, M. E. McIntyre, A. R. Douglass, R. B. Rood, and L. Pfister, 1995: Stratosphere-troposphere exchange. Rev. Geophys., 33, 403-439, https://doi.org/10.1029/ 95RG02097.

Hu, J.-G., R.-C. Ren, and H.-M. Xu, 2014: Occurrence of winter stratospheric sudden warming events and the seasonal timing of spring stratospheric final warming. J. Atmos. Sci., 71, 23192334, https://doi.org/10.1175/JAS-D-13-0349.1.

Huang, N. E., and S. S. P. Shen, Eds., 2005: Hilbert-Huang Transform and Its Applications. World Scientific, $311 \mathrm{pp}$. , and $\mathrm{Z}$. Wu, 2008: A review on Hilbert-Huang transform: Method and its applications to geophysical studies. Rev. Geophys., 46, RG2006, https://doi.org/10.1029/ 2007RG000228.

— noise-assisted data analysis method. Adv. Adapt. Data Anal., 1, 1-41, https://doi.org/10.1142/S1793536909000047.

_ method and the Hilbert spectrum for nonlinear and nonstationary time series analysis. Proc. Roy. Soc. London, 454A, 903-995, https://doi.org/10.1098/rspa.1998.0193.

_ Z Z. Shen, and S. R. Long, 1999: A new view of nonlinear water waves: The Hilbert spectrum. Annu. Rev. Fluid Mech., 31, 417-457, https://doi.org/10.1146/annurev.fluid.31.1.417.

Ji, F., Z. Wu, J. Huang, and E. P. Chassignet, 2014: Evolution of land surface air temperature trend. Nat. Climate Change, $\mathbf{4}$, 462-466, https://doi.org/10.1038/nclimate2223.

Johnson, D. R., 1989: The forcing and maintenance of global monsoonal circulations: An isentropic analysis. Advances in Geophysics, Vol. 31, Academic Press, 43-316, https://doi.org/ 10.1016/S0065-2687(08)60053-9.

Kenyon, J., and G. C. Hegerl, 2008: Influence of modes of climate variability on global temperature extremes. J. Climate, 21, 3872-3889, https://doi.org/10.1175/2008JCLI2125.1.

Kidston, J., A. A. Scaife, S. C. Hardiman, D. M. Mitchell, N. Butchart, M. P. Baldwin, and L. J. Gray, 2015: Stratospheric influence on tropospheric jet streams, storm tracks and surface weather. Nat. Geosci., 8, 433-440, https://doi.org/10.1038/ ngeo2424. 
Kodera, K., K. Yamazaki, M. Chiba, and K. Shibata, 1990: Downward propagation of upper stratospheric mean zonal wind perturbation to the troposphere. Geophys. Res. Lett., 17, 1263-1266, https://doi.org/10.1029/GL017i009p01263.

- , H. Mukougawa, and S. Itoh, 2008: Tropospheric impact of reflected planetary waves from the stratosphere. Geophys. Res. Lett., 35, L16806, https://doi.org/10.1029/2008GL034575.

Kolstad, E. W., T. Breiteig, and A. A. Scaife, 2010: The association between stratospheric weak polar vortex events and cold air outbreaks in the Northern Hemisphere. Quart. J. Roy. Meteor. Soc., 136, 886-893, https://doi.org/10.1002/qj.620.

Kuroda, Y., and K. Kodera, 1999: Role of planetary waves in the stratosphere-troposphere coupled variability in the Northern Hemisphere winter. Geophys. Res. Lett., 26, 2375-2378, https://doi.org/10.1029/1999GL900507.

Kushner, P. J., and L. M. Polvani, 2004: Stratosphere-troposphere coupling in a relatively simple AGCM: The role of eddies. J. Climate, 17, 629-639, https://doi.org/10.1175/1520-0442 (2004)017<0629:SCIARS >2.0.CO;2.

Kuttippurath, J., and G. Nikulin, 2012: A comparative study of the major sudden stratospheric warmings in the Arctic winters 2003/2004-2009/2010. Atmos. Chem. Phys., 12, 8115-8129, https://doi.org/10.5194/acp-12-8115-2012.

Lehtonen, I., and A. Y. Karpechko, 2016: Observed and modeled tropospheric cold anomalies associated with sudden stratospheric warmings. J. Geophys. Res. Atmos., 121, 1591-1610, https://doi.org/10.1002/2015JD023860.

Li, J.-P., and R.-Q. Ding, 2011: Temporal-spatial distribution of atmospheric predictability limit by local dynamical analogs. Mon. Wea. Rev., 139, 3265-3283, https://doi.org/10.1175/ MWR-D-10-05020.1.

Liberato, M. L. R., J. M. Castanheira, L. de la Torre, C. C. DaCamara, and L. Gimeno, 2007: Wave energy associated with the variability of the stratospheric polar vortex. J. Atmos. Sci., 64, 2683-2694, https://doi.org/10.1175/JAS3978.1.

Limpasuvan, V., D. W. J. Thompson, and D. L. Hartmann, 2004: The life cycle of the Northern Hemisphere sudden stratospheric warmings. J. Climate, 17, 2584-2596, https://doi.org/ 10.1175/1520-0442(2004)017<2584:TLCOTN>2.0.CO;2.

— D. L. Hartmann, D. W. J. Thompson, K. Jeev, and Y. L. Yung, 2005: Stratosphere-troposphere evolution during polar vortex intensification. J. Geophys. Res., 110, D24101, https:// doi.org/10.1029/2005JD006302.

Martius, O., L. M. Polvani, and H. C. Davies, 2009: Blocking precursors to stratospheric sudden warming events. Geophys. Res. Lett., 36, L14806, https://doi.org/10.1029/2009GL038776.

Matsuno, T., 1970: Vertical propagation of stationary planetary waves in the winter Northern Hemisphere. J. Atmos. Sci., 27, 871-883, https://doi.org/10.1175/1520-0469(1970)027<0871: VPOSPW $>2.0 . \mathrm{CO} ; 2$.

Matthewman, N., J. Esler, A. Charlton-Perez, and L. Polvani, 2009: A new look at stratospheric sudden warmings. Part III: Polar vortex evolution and vertical structure. J. Climate, 22, 15661585, https://doi.org/10.1175/2008JCLI2365.1.

McDaniel, B. A., and R. X. Black, 2005: Intraseasonal dynamical evolution of the northern annular mode. J. Climate, 18, 38203839, https://doi.org/10.1175/JCLI3467.1.

Mitchell, D. M., A. Charlton-Perez, and L. Gray, 2011: Characterizing the variability and extremes of the stratospheric polar vortices using 2D moment analysis. J. Atmos. Sci., 68, 1194 1213, https://doi.org/10.1175/2010JAS3555.1.

_ L. J. Gray, J. Anstey, M. P. Baldwin, and A. J. Charlton-Perez, 2013: The influence of stratospheric vortex displacements and splits on surface climate. J. Climate, 26, 2668-2682, https:// doi.org/10.1175/JCLI-D-12-00030.1.

Nishii, K., H. Nakamura, and Y. J. Orsolini, 2011: Geographical dependence observed in blocking high influence on the stratospheric variability through enhancement and suppression of upward planetary-wave propagation. J. Climate, 24, 6408-6423, https://doi.org/10.1175/JCLI-D-10-05021.1.

Palmeiro, F. M., D. Barriopedro, R. García-Herrera, and N. Calvo, 2015: Comparing sudden stratospheric warming definitions in reanalysis data. J. Climate, 28, 6823-6840, https://doi.org/ 10.1175/JCLI-D-15-0004.1.

Polvani, L. M., and D. W. Waugh, 2004: Upward wave activity flux as a precursor to extreme stratospheric events and subsequent anomalous surface weather regimes. J. Climate, 17, 3548-3554, https://doi.org/10.1175/1520-0442(2004)017<3548: UWAFAA $>2.0 . \mathrm{CO} ; 2$.

Seviour, W. J. M., D. M. Mitchell, and L. J. Gray, 2013: A practical method to identify displaced and split stratospheric polar vortex events. Geophys. Res. Lett., 40, 5268-5273, https:// doi.org/10.1002/grl.50927.

Shaw, T. A., and J. Perlwitz, 2013: The life cycle of Northern Hemisphere downward wave coupling between the stratosphere and troposphere. J. Climate, 26, 1745-1763, https:// doi.org/10.1175/JCLI-D-12-00251.1.

_, and - 2014: On the control of the residual circulation and stratospheric temperatures in the Arctic by planetary wave coupling. J. Atmos. Sci., 71, 195-206, https://doi.org/10.1175/ JAS-D-13-0138.1.

Shepherd, T. G., 2002: Issues in stratosphere-troposphere coupling. J. Meteor. Soc. Japan Ser. II, 80, 769-792, https://doi.org/ 10.2151/jmsj.80.769.

Simmons, A., S. Uppala, D. Dee, and S. Kobayashi, 2007: ERAInterim: New ECMWF reanalysis products from 1989 onwards. ECMWF Newsletter, No. 110, ECMWF, Reading, United Kingdom, 25-35, https://doi.org/10.21957/pocnex23c6.

Stan, C., and D. M. Straus, 2009: Stratospheric predictability and sudden stratospheric warming events. J. Geophys. Res., 114, D12103, https://doi.org/10.1029/2008JD011277.

Thompson, D. W. J., M. P. Baldwin, and J. M. Wallace, 2002: Stratospheric connection to Northern Hemisphere wintertime weather: Implications for prediction. J. Climate, $\mathbf{1 5}$, 1421-1428, https://doi.org/10.1175/1520-0442(2002)015<1421: SCTNHW $>2.0 . C O ; 2$.

Tomassini, L., E. P. Gerber, M. Baldwin, F. Bunzel, and M. Giorgetta, 2012: The role of stratosphere-troposphere coupling in the occurrence of extreme winter cold spells over northern Europe. J. Adv. Model. Earth Syst., 4, M00A03, https://doi.org/10.1029/2012MS000177.

Tripathi, O. P., and Coauthors, 2015: The predictability of the extratropical stratosphere on monthly time-scales and its impact on the skill of tropospheric forecasts. Quart. J. Roy. Meteor. Soc., 141, 987-1003, https://doi.org/10.1002/qj.2432.

Woo, S.-H., B.-M. Kim, and J.-S. Kug, 2015: Temperature variation over East Asia during the lifecycle of weak stratospheric polar vortex. J. Climate, 28, 5857-5872, https://doi.org/10.1175/ JCLI-D-14-00790.1.

Wu, Z., N. E. Huang, and X. Chen, 2009: The multi-dimensional ensemble empirical mode decomposition method. $A d v$. Adapt. Data Anal., 1, 339-372, https://doi.org/10.1142/ S1793536909000187.

Yoden, S., K. Ishioka, D. Durran, T. Enomoto, Y.-Y. Hayashi, T. Miyoshi, and M. Yamada, 2014: Theoretical aspects of variability and predictability in weather and climate systems. 
Bull. Amer. Meteor. Soc., 95, 1101-1104, https://doi.org/ 10.1175/BAMS-D-14-00009.1.

Yu, Y.-Y., M. Cai, R.-C. Ren, and H. M. van den Dool, 2015a: Relationship between warm airmass transport into upper polar atmosphere and cold air outbreaks in winter. J. Atmos. Sci., 72, 349-368, https://doi.org/10.1175/JAS-D14-0111.1.

- R.-C. Ren, and M. Cai, 2015b: Dynamical linkage between cold air outbreaks and intensity variations of the meridional mass circulation. J. Atmos. Sci., 72, 3214-3232, https://doi.org/ 10.1175/JAS-D-14-0390.1.

,-- , and $-2015 \mathrm{c}$ : Comparison of the mass circulation and $\mathrm{AO}$ indices as indicators of cold air outbreaks in northern winter. Geophys. Res. Lett., 42, 2442-2448, https://doi.org/ 10.1002/2015GL063676.

- M. Cai, and R.-C. Ren, 2018a: A stochastic model with a lowfrequency amplification feedback for the stratospheric northern annular mode. Climate Dyn., 50, 3757-3773, https:// doi.org/10.1007/s00382-017-3843-2.

,,--- , and J. Rao, 2018b: A closer look at the relationships between meridional mass circulation pulses in the stratosphere and cold air outbreak patterns in Northern Hemispheric winter. Climate Dyn., https://doi.org/10.1007/s00382-018-4069-7, in press. Zhang, Q., C.-S. Shin, H. M. van den Dool, and M. Cai, 2013: CFSv2 prediction skill of stratospheric temperature anomalies. Climate Dyn., 41, 2231-2249, https://doi.org/10.1007/s00382-013-1907-5. 